\title{
Gata2-L359V impairs primitive and definitive hematopoiesis and blocks cell differentiation in murine chronic myelogenous leukemia model
}

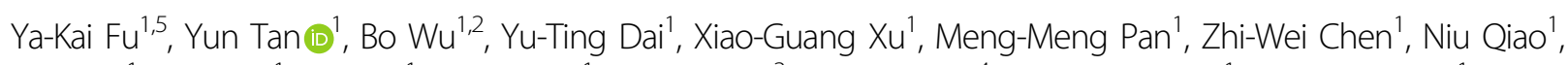

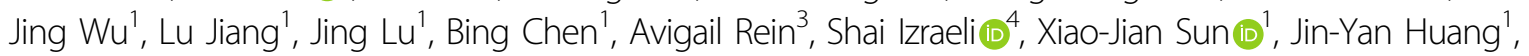
Qiu-Hua Huang (1)', Zhu Chen ${ }^{1}$ and Sai-Juan Chen (1)

\begin{abstract}
GATA2, a key transcription factor in hematopoiesis, is frequently mutated in hematopoietic malignancies. How the GATA2 mutants contribute to hematopoiesis and malignant transformation remains largely unexplored. Here, we report that Gata2-L359V mutation impeded hematopoietic differentiation in murine embryonic and adult hematopoiesis and blocked murine chronic myeloid leukemia (CML) cell differentiation. We established a Gata2-L359V knockin mouse model in which the homozygous Gata2-L359V mutation caused major defects in primitive erythropoiesis with an accumulation of erythroid precursors and severe anemia, leading to embryonic lethality around E11.5. During adult life, the Gata2-L359V heterozygous mice exhibited a notable decrease in bone marrow (BM) recovery under stress induction with cytotoxic drug 5-fluorouracil. Using RNA sequencing, it was revealed that homozygous Gata2-L359V suppressed genes related to embryonic hematopoiesis in yolk sac, while heterozygous Gata2-L359V dysregulated genes related to cell cycle and proliferation in BM Lin'Sca1 ${ }^{+}{ }^{C}-{ }^{-k i t}{ }^{+}$cells. Furthermore, through chromatin immunoprecipitation sequencing and transactivation experiments, we found that this mutation enhanced the DNA-binding capacity and transcriptional activities of Gata2, which was likely associated with the altered expression of some essential genes during embryonic and adult hematopoiesis. In mice model harboring $B C R /$ $A B L$, single-cell RNA-sequencing demonstrated that Gata2-L359V induced additional gene expression profile abnormalities and partially affected cell differentiation at the early stage of myelomonocytic lineage, evidenced by the increase of granulocyte-monocyte progenitors and monocytosis. Taken together, our study unveiled that Gata2-L359V mutation induces defective hematopoietic development and blocks the differentiation of CML cells.
\end{abstract}

Correspondence: Yun Tan (ty12260@rjh.com.cn) or

Qiu-Hua Huang (hqh10632@rih.com.cn) or Zhu Chen (zchen@stn.sh.cn) or Sai-Juan Chen (sjchen@stn.sh.cn)

'Shanghai Institute of Hematology, State Key Laboratory of Medical Genomics, National Research Center for Translational Medicine, Ruijin Hospital Affiliated to Shanghai Jiao Tong University (SJTU) School of Medicine, Shanghai, China ${ }^{2}$ Institute of Health Sciences, Shanghai Institutes for Biological Sciences and Graduate School, Chinese Academy of Sciences and SJTU School of Medicine, Shanghai, China

Full list of author information is available at the end of the article

These authors contributed equally: Ya-Kai Fu, Yun Tan, Bo Wu

Edited by M. Herold

\section{Introduction}

Hematopoietic differentiation is orchestrated by precise transcription programs and epigenetic regulation in distinct stages, and the dysregulation of key transcription and/or epigenetic factors may induce hematopoietic failure or malignant transformation ${ }^{1,2}$. High-throughput sequencing can be useful to identify malignancy-related mutations in these regulators ${ }^{3}$. However, the function of mutated regulators in normal and malignant hematopoiesis remains unexplored in many instances.

GATA2, a key transcription factor determining the differentiation/self-renewal fate of hematopoietic stem

\section{(c) The Author(s) 2021}

(c) (i) Open Access This article is licensed under a Creative Commons Attribution 4.0 International License, which permits use, sharing, adaptation, distribution and reproduction cc) in any medium or format, as long as you give appropriate credit to the original author(s) and the source, provide a link to the Creative Commons license, and indicate if changes were made. The images or other third party material in this article are included in the article's Creative Commons license, unless indicated otherwise in a credit line to the material. If material is not included in the article's Creative Commons license and your intended use is not permitted by statutory regulation or exceeds the permitted use, you will need to obtain permission directly from the copyright holder. To view a copy of this license, visit http://creativecommons.org/licenses/by/4.0/. 
cells (HSCs), also acts as one of the core regulators in erythrocytic differentiation ${ }^{4,5}$. The complete knockout (KO) of murine Gata2 results in hematopoietic failure and embryonic lethality ${ }^{6,7}$. Embryonic stem cells lacking Gata2 fail to undergo definitive hematopoiesis and exhibit defects in the production of all hematopoietic lineages ${ }^{6,8,9}$. Deleting the enhancer of Gata2 also impairs the selfrenewal of HSC and leads to impeded differentiation of erythropoietic progenitors in murine embryos, indicative of sufficient expression of Gata2 being required for the embryonic erythropoiesis ${ }^{10}$. Intriguingly, the ectopic overexpression of Gata2 reduces the expression of cell cycle-related genes, such as $C D K 4$ and $C D K 6$, facilitating the quiescence of $\mathrm{HSCs}^{11,12}$. Maintaining the accurate expression and function of Gata2 is thus pivotal for normal hematopoietic differentiation.

GATA2 is frequently mutated in hematopoietic malignancies, including acute erythroid leukemia ${ }^{13,14}$, acute myeloid leukemia (AML), and myelodysplastic syndrome $(\mathrm{MDS})^{15,16}$. Aberrant GATA2 expression is associated with poor clinical outcomes in MDS and AML ${ }^{17,18}$. Most
GATA2 mutations occur in the GATA2 DNA-binding domains such as the zinc-finger domains 1 (ZF1) and 2 (ZF2), and these mutations are believed to affect the transcriptional function of GATA $2^{19}$. By comparing the DNA-binding affinity with wild-type (WT) GATA2, GATA2 mutations are generally classified as "loss-offunction" (such as A318T, G320V, and T358N) or "gainof-function" ones (such as L359V) ${ }^{20}$. Both functional forms of mutations can be found in hematopoietic malignancies, assumedly disrupting the hematopoietic differentiation of HSC and progenitor cells ${ }^{21}$, but the in vivo functions of these mutations are rarely reported and need further study.

GATA2-L359V mutation, initially identified in patients with myelomonocytic transformation of chronic myelogenous leukemia $(\mathrm{CML})^{22}$, also exists in patients with AML and is associated with poor prognosis ${ }^{16,23}$. The preliminary in vitro study showed that this mutation could not only increase the DNA-binding capacity and the transcriptional activity of GATA2 but also enhance its inhibitory effect on PU.1, an important regulator of

A

Gata2

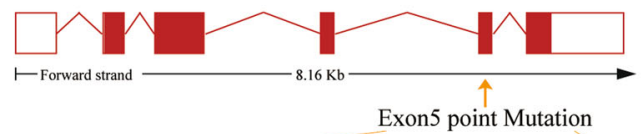

Wild-type mucleotide sequence

GCAAATTGTCAGACGACAACCACCACCTTATGGCGC

Mutated nucleotide sequence

GCAAATTGTCAGACGACAACCACCACCGTATGGCGC

\section{B}

E9.5

Gata2 wT/wT
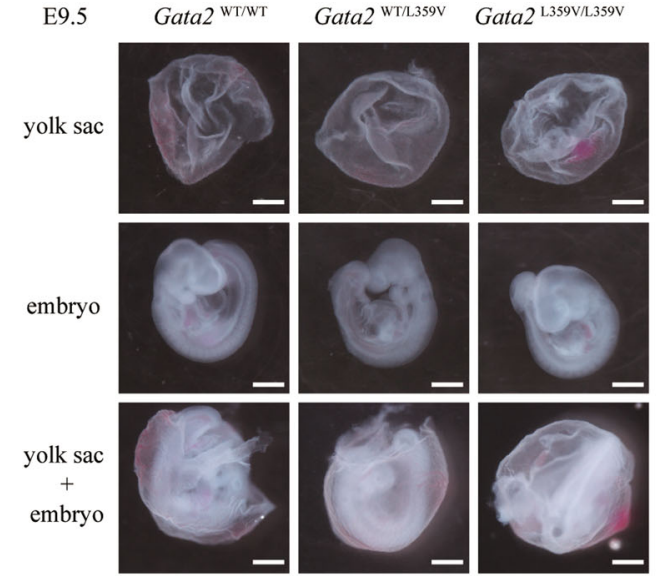
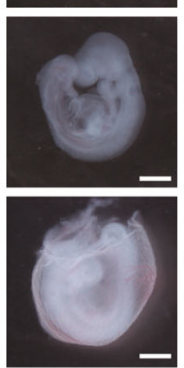

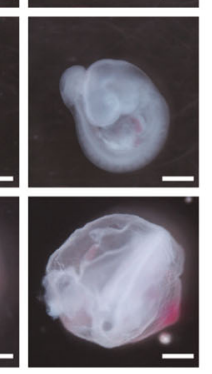

embryo

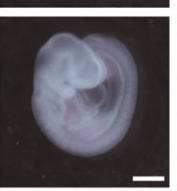

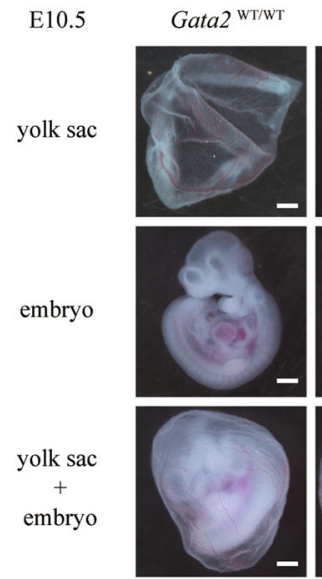

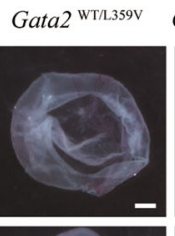

Gata2 $2^{\text {L359V/L359V }}$

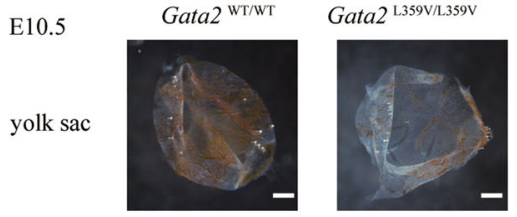

C

Fig. 1 Anemia phenotype in Gata2 ${ }^{\text {L359V/L359V }}$ embryos. A Schematic of the Gata2-L359V knockin murine model. The L359V mutation was introduced into the ZF2, which is located in the exon 5 of the murine Gata2 gene. B Morphology of embryos and yolk sacs of different genotypes at E9.5 (left panel, scale bar $500 \mu \mathrm{m}$ ) and E10.5 (right panel, scale bar $500 \mu \mathrm{m}$ ). C O-dianisidine staining of hemoglobin in yolk sac erythrocytes (scale bar $500 \mu \mathrm{m})$. 


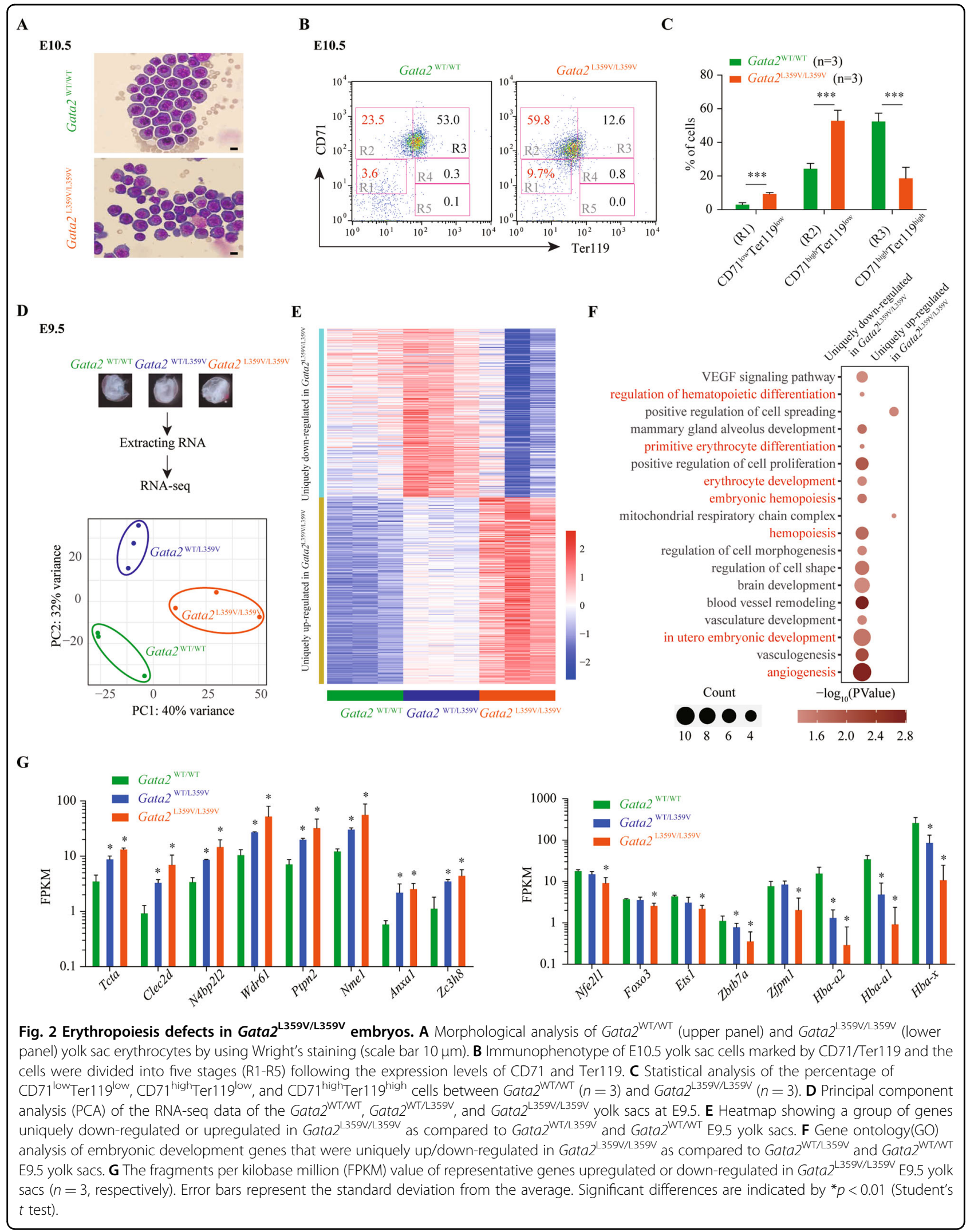


myelopoiesis $^{16,21,22}$. However, the functions of such mutations in vivo, particularly the underlying regulatory mechanism in hematopoiesis and malignant transformation, remain largely unknown.

In the current study, we established a Gata2-L359V mutation knockin mouse model, intending to examine the effect of this mutation on the murine hematopoiesis and the differentiation block of CML cells.

\section{Results}

\section{Homozygous Gata2-L359V mutation is associated with embryonic lethality}

To investigate the pathophysiological roles of Gata2L359V mutation in vivo, we generated a Gata2-L359V knockin murine model (Fig. 1a and S1A, B). No homozygous $\left(\mathrm{Gata}^{\mathrm{L} 359 \mathrm{~V} / \mathrm{L} 359 \mathrm{~V}}\right)$ mutant was detected among over 20 littermates of heterozygous (Gata2 ${ }^{\mathrm{WT} / \mathrm{L} 359 \mathrm{~V}}$ ) intercrosses (Figure S1C and Table S1), suggesting that Gata $2^{\mathrm{L} 359 \mathrm{~V} / \mathrm{L} 359 \mathrm{~V}}$ mutants were embryonically lethal. The genotype of the embryo was identified by genomic DNA PCR. The time of homozygous embryonic death was determined using embryos from timed matings (Fig. S1D). At embryonic day (E) 10.5, all the genotypes of surviving embryos showed the expected Mendelian frequency. Besides, we also observed the genetic knockin did not alter the expression levels of WT or mutant Gata2 protein in embryos (Fig. S1E). However, no Gata $2^{\mathrm{L} 359 \mathrm{~V} / \mathrm{L} 359 \mathrm{~V}}$ embryo survived beyond E11.5, whereas Gata2 $2^{\mathrm{WT} / \mathrm{WT}}$ and Gata2 ${ }^{\mathrm{WT} / \mathrm{L} 359 \mathrm{~V}}$ embryos remained viable at all embryonic stages (Table S1). These observations indicated that homozygous Gata2-L359V mutation resulted in midgestational embryonic lethality.

Next, we analyzed the embryos and yolk sacs, no morphological difference was observed among Gata $2^{\mathrm{L} 359 \mathrm{~V} / \mathrm{L} 359 \mathrm{~V}}$, Gata $2^{\mathrm{WT} / \mathrm{L} 359 \mathrm{~V}}$, and Gata $2^{\mathrm{WT} / \mathrm{WT}}$ mutants at E9.5. However, Gata $2^{\mathrm{L} 359 \mathrm{~V} / \mathrm{L} 359 \mathrm{~V}}$ embryos, which presented with pale yolk sacs, showed growth retardation at E10.5 (Fig. 1b). The o-dianisidine staining showed considerably decreased hemoglobin levels in Gata $2^{\mathrm{L} 359 \mathrm{~V} / \mathrm{L} 359 \mathrm{~V}}$ yolk sacs at E10.5 (Fig. 1c), reflecting a decrease in erythropoiesis. These results indicated that homozygous mutation of Gata2L359V induced severe anemia during mouse embryonic development.

\section{Homozygous Gata2-L359V mutation impairs embryonic erythroid differentiation}

To investigate the mechanism underlying anemia in Gata $2^{\mathrm{L} 359 \mathrm{~V} / \mathrm{L} 359 \mathrm{~V}}$ yolk sacs, we determined the morphological features and immunophenotypes of Gata $2^{\mathrm{L} 359 \mathrm{~V} / \mathrm{L} 359 \mathrm{~V}}$ and Gata2 ${ }^{\mathrm{WT} / \mathrm{WT}}$ yolk sacs. Approximately $70 \%$ of the erythrocytes in E10.5 Gata $2^{\mathrm{L} 359 \mathrm{~V} / \mathrm{L} 359 \mathrm{~V}}$ yolk sacs exhibited increased nucleus-to-cytoplasm ratio and dark bluestained cytoplasm (Fig. 2a), suggesting impaired development of primitive erythropoiesis. We then performed immunophenotyping analysis by using antibodies against CD71 and Ter119. The embryonic erythroid development can be divided into five stages (designated as R1-R5), corresponding to different stages during erythroid differentiation $^{24}$ (Fig. 2b). Gata2 $2^{\mathrm{L} 359 \mathrm{~V} / \mathrm{L} 359 \mathrm{~V}}$ mutants showed significantly increased CD71 $1^{\text {low }}$ Ter $119^{\text {low }}$ and CD71 ${ }^{\text {high }}$ Ter119 $9^{\text {low }}$ precursors (R1 and R2 compartments) and decreased CD71 ${ }^{\text {high }}$ Ter119 ${ }^{\text {high }}$ cells (R3 compartment; Figs. 2c and S2), indicating that homozygous Gata2-L359V mutation blocked embryonic erythropoiesis. Compared with erythroid marker CD71, the expression of myeloid markers and transcription factors were extremely low (Fig. S3A).

To further determine the program dysregulated by Gata2-L359V mutation during embryonic erythropoiesis, we performed RNA sequencing (RNA-seq) analysis in Gata2 $^{\mathrm{L} 359 \mathrm{~V} / \mathrm{L} 359 \mathrm{~V}}$, Gata2 ${ }^{\mathrm{WT} / \mathrm{L} 359 \mathrm{~V}}$, and Gata2 ${ }^{\mathrm{WT} / \mathrm{WT}}$ yolk sacs at E9.5 (Fig. 2d). A group of genes that were uniquely up/down-regulated in Gata2 $2^{\mathrm{L} 359 \mathrm{~V} / \mathrm{L} 359 \mathrm{~V}}$ as compared to Gata2 $^{\mathrm{WT} / \mathrm{L} 359 \mathrm{~V}}$ and Gata2 ${ }^{\mathrm{WT} / \mathrm{WT}}$ E9.5 yolk sacs were identified (Fig. 2e). With Gene ontology (GO) analysis, these genes were largely of functional relevance to embryonic hematopoiesis (e.g., erythrocyte development, hematopoietic progenitor cell differentiation, and primitive erythrocyte differentiation) and regulation of vascular development (e.g., vasculature development, angiogenesis, VEGF signaling pathway, blood vessel remodeling; Fig. 2f and Table S2). As shown in Fig. 2g, the expression levels of genes annotated for hematopoietic differentiation ${ }^{25,26}$, such as $N f e 2 l 1^{27}, E t s 1^{28}, Z b t b 7 a^{29}$, and $Z f p m 1^{30}$, were inhibited in Gata $2^{\mathrm{L} 359 \mathrm{~V} / \mathrm{L} 359 \mathrm{~V}}$ group. Meanwhile, Hba-a2, $H b a-a 1$, and $H b a-x$ were down-regulated in Gata $2^{\mathrm{L} 359 \mathrm{~V} / \mathrm{L} 359 \mathrm{~V}}$ sample, which was consistent with the anemia phenotype during the embryonic development (Figs. $2 \mathrm{~g}$ and S3B). Additionally, we found some genes up/down-regulated in both Gata2 $2^{\mathrm{L} 359 \mathrm{~V} / \mathrm{L} 359 \mathrm{~V}}$ and Gata2 $2^{\mathrm{WT} / \mathrm{L} 359 \mathrm{~V}}$ as compared to Gata2 ${ }^{\text {WT/WT }}$ group. GO analysis showed that genes related to certain metabolic pathways were upregulated, while those related to some important signal transduction pathways (such as Notch and Wnt pathways) were downregulated in the two former groups (Fig. S3C), implying that although Gata2 $2^{\mathrm{WT} / \mathrm{L} 359 \mathrm{~V}}$ mutants escaped from embryonic lethality, they might still carry subtle defects of physiological function due to the abnormal gene expression.

\section{Heterozygous Gata2-L359V mutation significantly affects hematopoietic reconstitution}

Gata $2^{\mathrm{L} 359 \mathrm{~V} / \mathrm{L} 359 \mathrm{~V}}$ mutants died around E11.5, but Gata2 $^{\mathrm{WT} / \mathrm{L} 359 \mathrm{~V}}$ mice remained viable. The peripheral blood (PB) cellular components, bone marrow (BM) cell morphological features, immunophenotypes, and frequencies of hematopoietic stem/progenitor cell (HSPC) subsets appeared to be normal (Fig. S4A-E), and the 


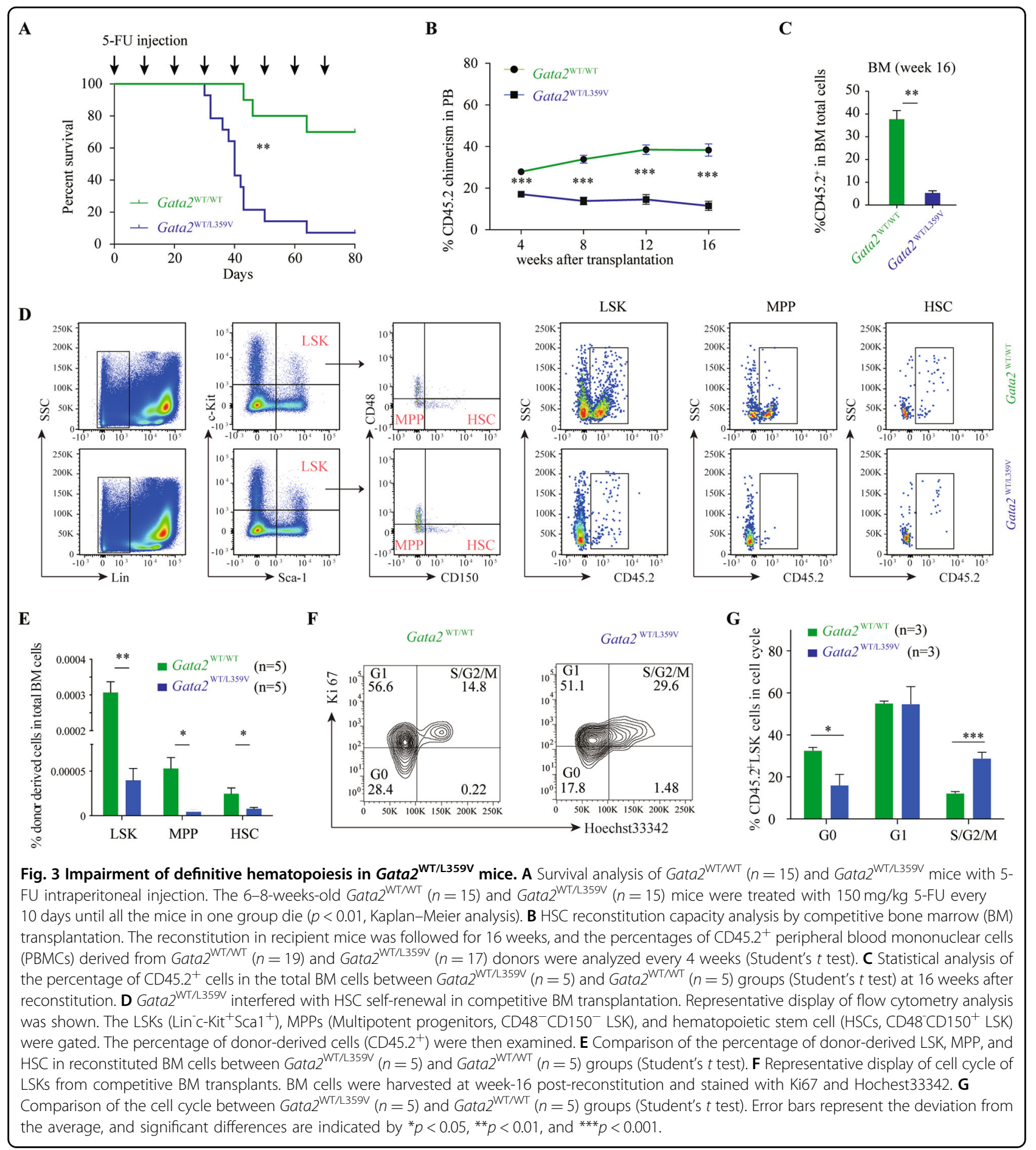

colony-forming capacities between Gata2 $2^{\mathrm{WT} / \mathrm{WT}}$ and Gata2 ${ }^{\mathrm{WT} / \mathrm{L} 359 \mathrm{~V}}$ BM cells showed no significant differences (Fig. S4F).

To have an in-depth investigation on the role of Gata2L359V mutation in adult hematopoiesis, we treated Gata $2^{\mathrm{WT} / \mathrm{L} 359 \mathrm{~V}}$ and Gata2 ${ }^{\mathrm{WT} / \mathrm{WT}}$ mice with the cytotoxic drug 5-fluorouracil (5-FU) to examine the effect of this mutation on the hematopoietic recovery under stress condition $^{31,32}$. The result showed that over $92.8 \%$ of Gata $2^{\mathrm{WT} / \mathrm{L} 359 \mathrm{~V}}$ mice died, whereas only $30 \%$ of Gata $2^{\mathrm{WT} / \mathrm{WT}}$ mice succumbed (Fig. 3a). We then performed competitive $\mathrm{BM}$ transplantation experiments to clarify whether Gata2-L359V mutation could interfere with HSC selfrenewal. BM cells of the donor (CD45.2 Gata2 $2^{\mathrm{WT} / \mathrm{WT}}$ and 


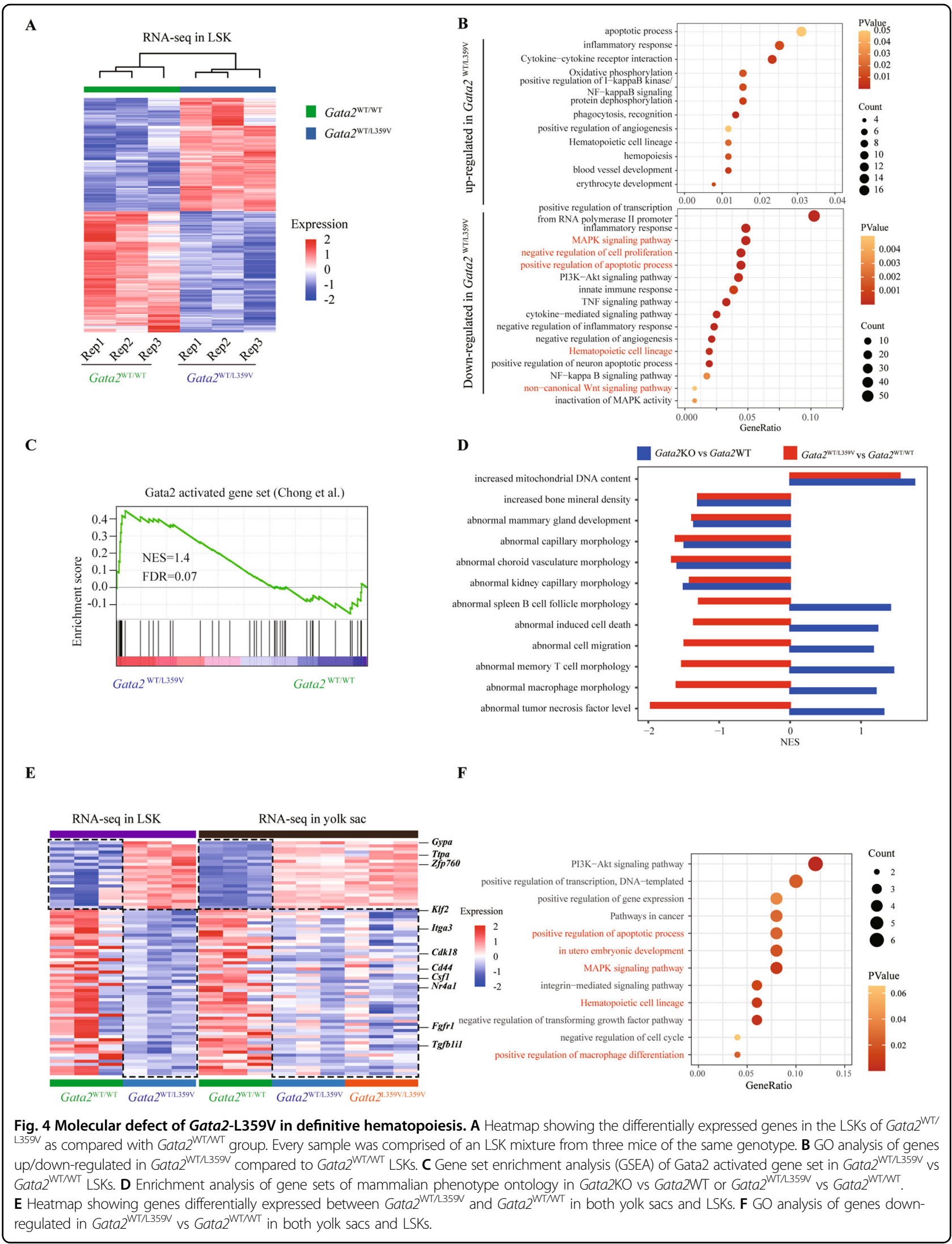


Gata $^{\mathrm{WT} / \mathrm{L} 359 \mathrm{~V}}$ ) and competitor mice (CD45.1) were mixed in the ratio of $1: 1$ and transplanted into recipient mice. Over the 16-week follow-up, the Gata $2^{\mathrm{WT} / \mathrm{WT}}$ donor-derived cells increased from $27.89 \%$ to $38.32 \%$ in $\mathrm{PB}$, whereas Gata2 ${ }^{\mathrm{WT} / \mathrm{L} 359 \mathrm{~V}}$ donor-derived cells significantly decreased at all time points (from $17.07 \%$ to $11.50 \% ; P<0.01$, Fig. 3b). An obvious decrease of Gata $^{\text {WT/L359V }}$ donor-derived cells was also observed in BM (Fig. 3c). Moreover, immunophenotype analysis revealed a remarkable reduction in the frequency of Gata2 ${ }^{\mathrm{WT} / \mathrm{L} 359 \mathrm{~V}}$ donor-derived $\mathrm{CD} 45.2^{+} \mathrm{CD} 150^{+} \mathrm{CD} 48^{-}$ $\mathrm{Lin}^{-} \mathrm{Sca}-1^{+} \mathrm{c}-\mathrm{Kit}^{+}$(hematopoietic stem cells $[\mathrm{HSCs}]^{33}$ ) and $\mathrm{CD} 45.2^{+} \mathrm{CD} 150^{-} \mathrm{CD} 48^{-} \mathrm{Lin}^{-} \mathrm{Sca}-1^{+} \mathrm{c}-\mathrm{Kit}^{+}$cells (multipotent progenitors [MPPs], this marker combination being chosen to avoid discrepancy in the literation on the definition of $\mathrm{MPP}^{33-35}$ ) in recipients, indicating that Gata2-L359V mutated HSCs were impaired in hematopoietic reconstitution under stress condition (Fig. 3d, e). Apart from the lesion of HSCs and MPPs, the numbers of Gata $2^{\mathrm{WT} / \mathrm{L} 359 \mathrm{~V}}$ donor-derived downstream myeloid progenitors including common myeloid progenitor (CMP, $\left.\mathrm{Lin}^{-} \mathrm{Sca1}{ }^{-} \mathrm{C}-\mathrm{Kit}^{+} \mathrm{CD} 34^{+} \mathrm{CD} 16 / 32^{\text {high }}\right)$, granulocyte/ monocyte progenitor (GMP, $\mathrm{Lin}^{-} \mathrm{Scal}^{-} \mathrm{c}-\mathrm{Kit}^{+} \mathrm{CD} 34^{+}$ $\left.\mathrm{CD} 16 / 32^{\text {low }}\right)$, and megakaryocyte/erythroid progenitor

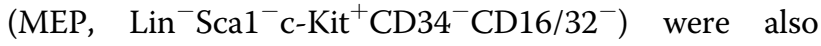
decreased (Fig. S5G). To better understand these reconstitution defects, we carried out cell cycle analysis on BM Lin ${ }^{-} \mathrm{Sca}-1^{+} \mathrm{c}-\mathrm{Kit}^{+}$(LSK) cells at week-16 post-transplantation. The percentage of Gata $2^{\mathrm{WT} / \mathrm{L} 359 \mathrm{~V}}$ donor-derived LSKs at G0 phase was dramatically decreased, whereas the percentage of that at $\mathrm{S} / \mathrm{G} 2 / \mathrm{M}$ phase was significantly increased compared with the control group (Fig. 3f, g), indicating the effect of Gata2-L359V on HSC exhaustion.

\section{Heterozygous Gata2-L359V mutation induces subtle molecular alteration in definitive hematopoiesis}

To explore the molecular mechanism underlying HSC defects, we performed the RNA-seq on BM LSKs from 8week-old Gata2 $2^{\mathrm{WT} / \mathrm{WT}}$ and Gata2 ${ }^{\mathrm{WT} / \mathrm{L} 359 \mathrm{~V}}$ mice under steady-state conditions (Table S3). Unsupervised hierarchical clustering revealed the difference in gene expression profiles between two groups (Fig. 4a). GO analysis showed that some genes related to negative regulation of cell cycle and proliferation (such as Klf4, Osm, and $N r 2 e 3$ ), positive regulation of apoptotic process (such as Tnf, Gadd45b, and Bcl211), Wnt signaling pathways (such as Wnt11, Fzd4, and $F z d 8$ ), and MAPK signaling pathway (such as Fgf3, Fos, and Hspa1b) were downregulated in the LSKs of Gata2 ${ }^{\mathrm{WT} / \mathrm{L} 359 \mathrm{~V}}$ as compared with those in Gata2 ${ }^{\mathrm{WT} / \mathrm{WT}}$ group (Fig. $4 \mathrm{~b}$ and Table S3). However, when some genes essential for HSC self-renewal such as $\operatorname{Spi1}^{36}, \operatorname{Tet}^{37}$, Dnmt $3 a^{38}$, and Mllt3a ${ }^{39}$ were examined, no significant difference were observed (Fig. S5A), which might be ascribed to the inability of heterozygous Gata2 to induce dramatically transcriptomic changes under steady status. This observation also supported the lack of significant difference of the HSC and lineage bias between Gata $2^{\mathrm{WT} / \mathrm{L} 359 \mathrm{~V}}$ and Gata2 ${ }^{\mathrm{WT} / \mathrm{WT}}$.

Furthermore, we combined our transcriptome dataset of Gata2 $2^{\mathrm{WT} / \mathrm{L} 359 \mathrm{~V}}$ with that of heterozygous Gata2KO LSKs reported previously ${ }^{21,22,40}$. Genes activated by Gata2 tended to be enriched in Gata $2^{\text {WT/L359V }}$ versus Gata2 $2^{\text {WT/WT }}$ LSKs $(F D R=0.07)^{21,22}$ (Fig. 4c). Gene set enrichment analysis (GSEA) showed that although heterozygous Gata2-L359V and Gata2KO had opposite effects on some pathways involved in cell function, they showed similar expression patterns in many other important pathways (Fig. 4d). Besides, it has been reported that Gata2 overexpression inhibited genes involved in angiogenesis and endothelial cell differentiation ${ }^{41}$. Consistent with this report, our GSEA analysis also showed that gene sets of angiogenesis and endothelial cell differentiation were significantly down-regulated in Gata $2^{\mathrm{WTT} / \mathrm{L} 359 \mathrm{~V}}$ LSKs (Fig. S5B). Taken together, our data suggested that Gata2L359V mutation exerts complex transcriptional regulatory function. In addition, we compared the RNA-seq data between BM LSKs and yolk sacs. Indeed, Gata2L359V down-regulated some genes related to hematopoietic cell lineage ${ }^{25,26}$ (such as Csf1, Itga3, and Cd44) and in utero embryonic development ${ }^{25,26}$ (such as Meg3, Syvn1, Klf2, and Fgfr1) in both BM LSKs and yolk sacs (Fig. 4e, f).

\section{Gata2-L359V acquires enhanced chromatin-binding ability and transcriptional activity}

To explore the mechanism underlying the Gata2L359V mutation-mediated gene dysregulation, we ectopically expressed Flag-Gata2-L359V and Flag-Gata2-WT in 32D cells ${ }^{42}$ (Fig. 5a), a murine myeloid precursor cell line, and performed chromatin immunoprecipitation sequencing (ChIP-seq). By this approach, we preliminarily identified 546 Gata2-WT- and 1483 Gata2-L359V-bound regions (Table S4), and most of Gata2-WT bound regions were also bound by Gata2-L359V (Fig. 5b). We then compared the binding signals of Gata2-L359V and Gata2WT and identified 74 Gata2-WT uniquely bound regions, 1011 Gata2-L359V uniquely bound regions, and 472 Gata2-WT and Gata2-L359V co-bound regions. Intriguingly, on the overlapping regions, the binding signals of Gata2-L359V were significantly higher than those of Gata2-WT (Fig. 5c). On the Gata2-L359V unique regions, nevertheless, Gata2-WT still had weak binding signals. This result implied that Gata2-L359V mutation might not alter the Gata2 targets but could significantly enhance the binding affinity on these targets.

We next compared the genomic distribution patterns of these regions and found that the overlapping and Gata2$\mathrm{L} 359 \mathrm{~V}$ unique regions were distributed in a similar way 


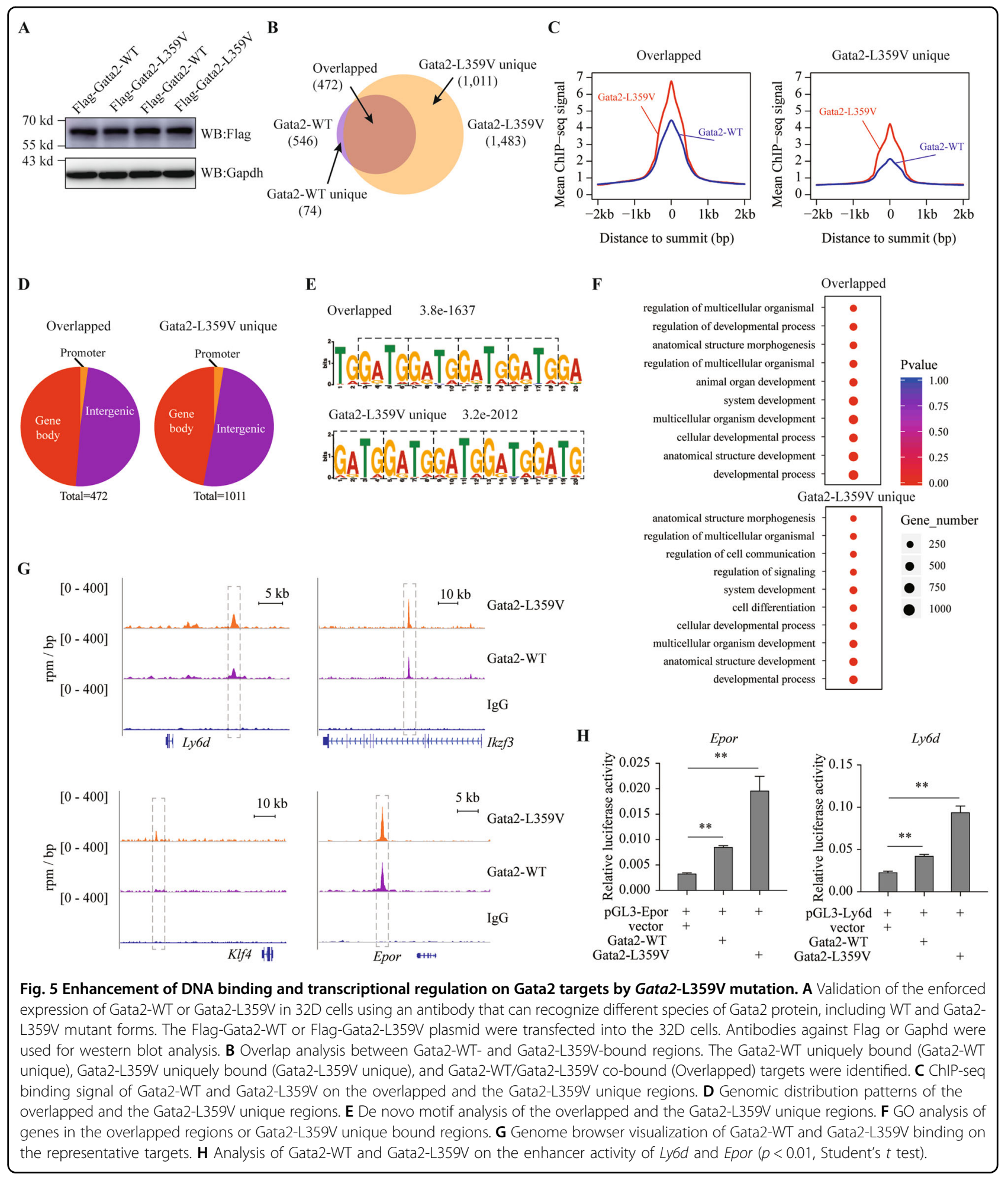

(Fig. 5d). Both WT and mutated Gata2 scattered widely across the whole genome, including promoter, gene body, and intergenic regions, as previously reported ${ }^{43}$. The motif analysis on the overlapping regions and the Gata2L359V unique regions showed that Gata2-L359V and
Gata2-WT both bound to the GATA/G repeat sequences (Fig. 5e). As previously reported, GATG motifs can also serve as binding sites for GATA2 besides the canonical GATA motifs ${ }^{44}$. Subsequently, we performed GO analysis of the genes in overlapping and Gata2-L359V unique 
A

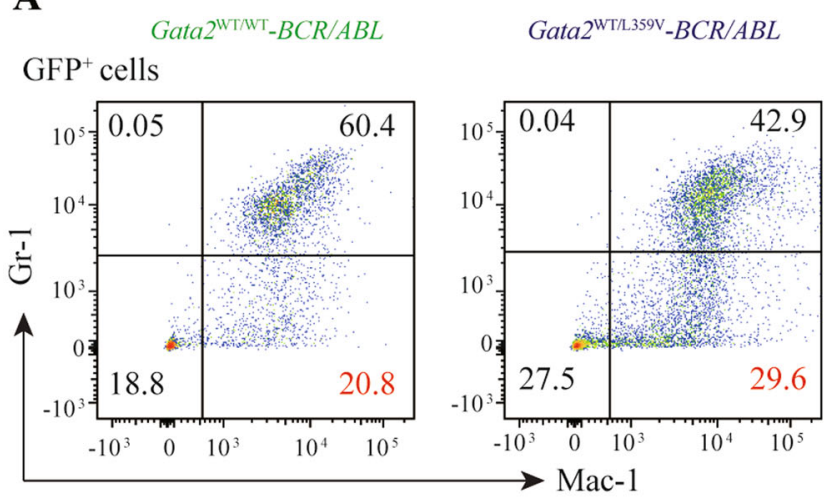

C

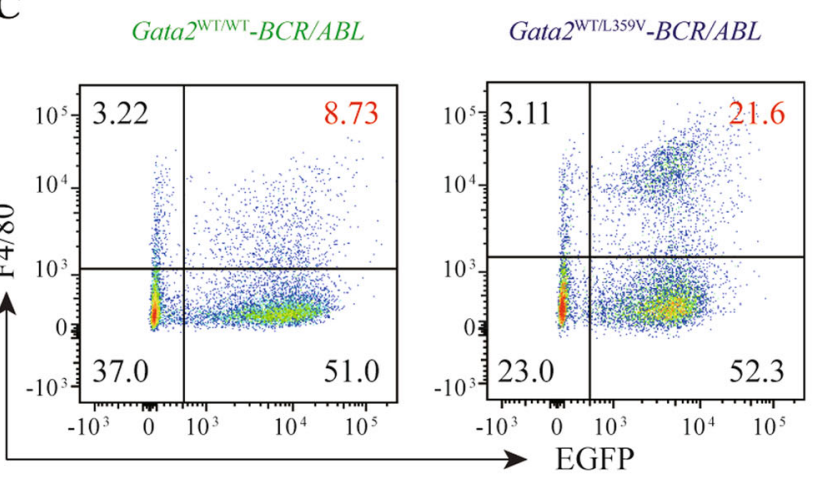

$\mathbf{E}$ Gata2 $^{\mathrm{WT} / N \mathrm{~T}}-B C R / A B L$
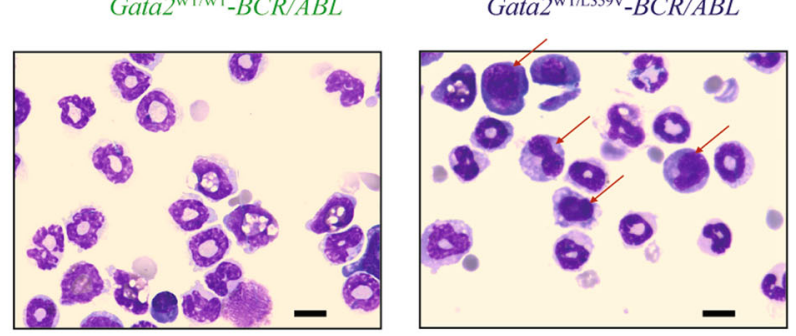

B

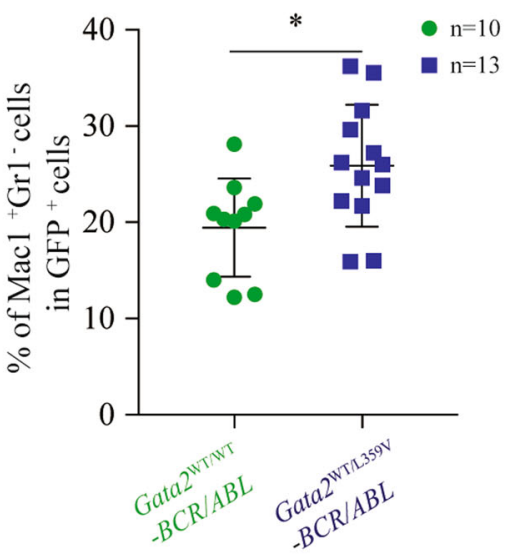

D

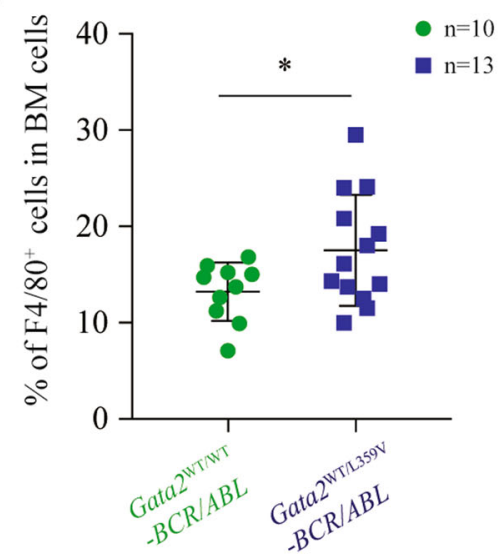

F

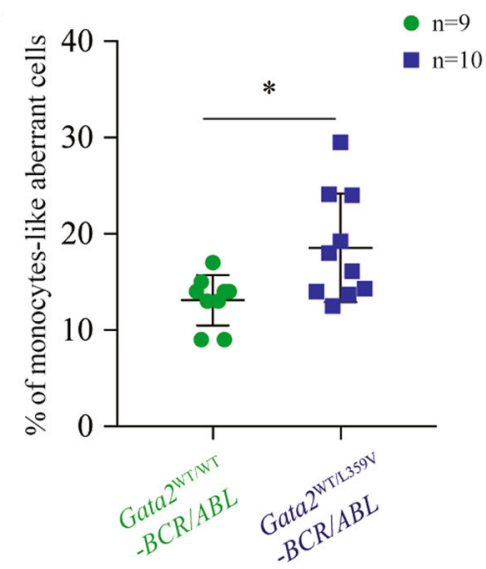

Fig. 6 Cell differentiation interference by Gata2-L359V mutation in a murine model of chronic myeloid leukemia. A Representative display of flow cytometry analysis of $\mathrm{Gr}_{-1}{ }^{-} \mathrm{Mac}^{-1}{ }^{+}$cells in $\mathrm{GFP}^{+} \mathrm{BM}$ cells. B Statistical analysis of $\mathrm{Gr}-1^{-} \mathrm{Mac}^{-1}{ }^{+}$cell frequencies between $\mathrm{Gata2}{ }^{\mathrm{WT} / \mathrm{WT}}-\mathrm{BCR} / \mathrm{ABL}$ $(n=10)$ and Gata $2^{\text {WT/L359V }}-B C R / A B L(n=13)$ mice. C Representative flow cytometry analysis of GFP ${ }^{+} F 4 / 80^{+}$cells in total BM cells. D Statistical analysis

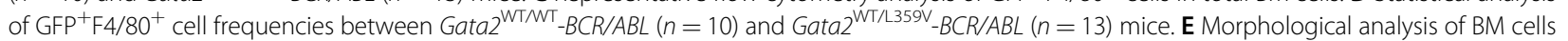
in Gata2 ${ }^{\text {WT } / W T}-B C R / A B L(n=10)$ and Gata2 ${ }^{\text {WT/L359V }}-B C R / A B L(n=13)$ mice. BM cells were harvested and subject to Wright's staining. The red arrows represent the atypical monocytes (scale bar $10 \mu \mathrm{m}$ ). $\mathbf{F}$ Statistical analysis of the percentage of monocyte-like aberrant cells in BM between Gata2 ${ }^{\text {WTMT }}$ $B C R / A B L(n=9)$ and Gata2 ${ }^{\text {WT/L359V }}-B C R / A B L(n=10)$ mice. Error bars represent the deviation from the average, and significant differences are indicated by ${ }^{*} p<0.05$ (Student's $t$-test). 
regions. It was found that a number of Gata2-L359V unique genes were related to cell differentiation (Fig. 5f), which might partially account for the abnormal hematopoietic differentiation in our model systems. The binding signals of Gata2-L359V and Gata2-WT on four representative targets were illustrated in Fig. 5g, including Epor (one of the master regulators of erythrocyte differentiation $^{41,45}$ ), Klf4, Ikzf3 (both known to be crucial for cell differentiation and stemness regulation ${ }^{46,47}$ ), and $L y 6 d$ (a specification marker of lineage commitment ${ }^{48}$ ). In line with the genome browser visualization, luciferase reporter assay showed that, though both Gata2-L359V and Gata2WT could activate the transcription of Epor and $L y 6 d$, the activation by Gata2-L359V was much stronger (Fig. 5h).

We also performed an integrative analysis of the ChIPseq and RNA-seq data. Amongst Gata2-L359V unique bound genes, Bcas3, Sall4, and Vegfa, annotated to embryonic lethality and abnormal hematopoiesis according to mammalian phenotype ontology ${ }^{26}$, showed different expression patterns between Gata2 $2^{\mathrm{L} 359 \mathrm{~V} / \mathrm{L} 359 \mathrm{~V}}$ and Gata2 ${ }^{\mathrm{WT} / \mathrm{L} 359 \mathrm{~V}}$ yolk sacs (Fig. S6A). As for Gata2-L359V unique bound genes in BM LSKs, hematopoietic transcription factor Klf4, Klf6, and Jun showed a significantly lower expression, while the expression of cell cycle-related gene Ccnd 1 was much increased, in Gata $2^{\mathrm{WT} / \mathrm{L} 359 \mathrm{~V}}$ group than in Gata $2^{\mathrm{WT} / \mathrm{WT}}$ one (Fig. S6B). These results implied that Gata2-L359V mutant might acquire the capacity to suppress the stemness of HSCs and promote cell cycle potentially through dysregulating the transcription of the key factors.

Meanwhile, we analyzed the overlap of the targeted genes between Gata2-L359V unique and previously reported GATA1/GATA2 datasets ${ }^{49}$. Amongst 2524 genes bound by both GATA1 and GATA2, 133 genes were overlapped with Gata2-L359V unique targets. GO analysis revealed that these genes were functionally involved in biological processes including cell differentiation, development, signal transduction, and metabolism (Fig. S6C). It implied that Gata2-L359V mutant might interfere with the GATA switch likely through these unique binding regions across the three settings.

\section{Gata2-L359V mutation disturbs the differentiation of $B C R /$ $A B L$-induced murine $\mathrm{CML}$ cells}

Considering that Gata2-L359V mutation was identified in the CML blast crisis ${ }^{22,50}$, we used a murine CML model to determine the role of this mutation in differentiation blockage. Gata $2^{\mathrm{WT} / \mathrm{WT}}$ and Gata2 $2^{\mathrm{WT} / \mathrm{L} 359 \mathrm{~V}}$ BM cells were infected with the $B C R / A B L$-expressing retrovirus and then injected into the lethally irradiated recipients ${ }^{51,52}$. The $B M$ immunophenotype analysis revealed a CML-like disease characterized by the massive expansion of mature granulocytes $\left(\mathrm{GFP}^{+} / \mathrm{Gr}-1^{+} / \mathrm{Mac}^{+} 1^{+}\right.$cells) in both groups (Fig. 6a). Notably, the number of $\mathrm{Gr}-1^{-} / \mathrm{Mac}-1^{+}$monocytic cells in the Gata2 $^{\mathrm{WT} / \mathrm{L} 359 \mathrm{~V}}-B C R / A B L$ group $(25.88 \% \pm 1.75 \%)$ was significantly higher than that in the Gata $2^{\mathrm{WT} / \mathrm{WT}}-B C R / A B L$ group $(19.44 \% \pm 1.61 \%$; $p=0.0158$; Fig. $6 \mathrm{~b})$. The $\mathrm{F} 4 / 80^{+}$ monocytic cells in Gata $2^{\mathrm{WT} / \mathrm{L} 359 \mathrm{~V}}-B C R / A B L$ mice $(17.51 \% \pm$ $1.60 \%)$ were also higher than those in Gata2 ${ }^{\mathrm{WT} / \mathrm{WT}}-B C R /$ $A B L$ mice $(13.21 \% \pm 0.95 \% ; p=0.0448$; Fig. 6 c, d). The $\mathrm{BM}$ cells of representative mice were collected and subjected to Wright's staining, showing the granulocytes significantly increased in both groups (Fig. 6e). However, in addition to the basic CML-like phenotype, a group of monocyte-like aberrant cells with irregularly shaped nuclei and dark blue cytoplasm was noticeable in the Gata $2^{\mathrm{WT} / \mathrm{L} 359 \mathrm{~V}}-B C R / A B L$ group (Fig. 6f). Similar to the previous findings ${ }^{51-53}$, Gata2 $2^{\text {WT/L359V }}-B C R / A B L$ mice showed a longer life span than Gata2 $^{\mathrm{WT} / \mathrm{WT}}-B C R / A B L$ mice (Fig. S7).

\section{Molecular signature alteration reveals the Gata2-L359V mutation as a driver for the increment of GMPs and monocytosis in $B C R / A B L$-induced CML model}

To compare the transcriptomic characteristics of BM cells between Gata $2^{\mathrm{WT} / \mathrm{L} 359 \mathrm{~V}}-B C R / A B L$ and Gata $2^{\mathrm{WT} / \mathrm{WT}}$ $-B C R / A B L$ mice, we performed single-cell RNA-seq (scRNA-seq) in two representative mice. A total of 7787 single cells were analyzed from which we identified 12 classes of cell types ${ }^{54,55}$ (Figs. 7a and S8A). Consistent with the murine CML phenotype, most of the BM cells in Gata $^{\text {WT/L359V }}-B C R / A B L$ and Gata2 ${ }^{\mathrm{WT} / \mathrm{WT}}-B C R / A B L$ mice were neutrophils $(\mathrm{C} 1, \mathrm{C} 3-\mathrm{C} 5$, and $\mathrm{C} 7)$. Monocytes, macrophages, erythroid progenitors, and GMPs were also noted. Three classes (C2, C6, and C12) of monocytes were characterized by $\operatorname{Itgam}^{\text {high }} \mathrm{Ly} \mathrm{gg}^{\text {low }}, \mathrm{Cd} 14^{\text {high }}$ Itgam $^{\text {high }}$, and F4/80(Adgre 1$)^{+} \mathrm{Csf1} 1 r^{+}$, respectively, and three classes (C8, $\mathrm{C} 9$, and C11) of GMPs were characterized by $\mathrm{Gata}^{+} \mathrm{Kit}^{+}$, $\mathrm{Ms} 4 \mathrm{a}^{+} \mathrm{Kit}^{+}$, and $\mathrm{Mpo}^{+} \mathrm{Kit}^{+}$, respectively, which represented distinct stages along with the monocytic differentiation ${ }^{56,57}$ (Fig. 7b, c). We then compared the ratio of each cell type in BM from Gata $2^{\mathrm{WT} / \mathrm{L} 359 \mathrm{~V}}-B C R / A B L$ or Gata $2^{\mathrm{WT} / \mathrm{WT}}-B C R / A B L$ mice and found that the three classes of monocytes and a small subgroup of erythrocytic progenitors $(\mathrm{C} 10)$ were increased in the former group. Notably, the percentages of C11, C9, and C8 GMPs were $0.4 \%, 3.9 \%$, and $4.0 \%$, respectively in the Gata $2^{\mathrm{WT} / \mathrm{WT}}$ $B C R / A B L$ vs $3.8 \%, 5.6 \%$, and $7.7 \%$, respectively in the Gata $2^{\mathrm{WT} / \mathrm{L} 359 \mathrm{~V}}-B C R / A B L$ group, indicating an increment of progenitors in the Gata2-L359V setting. Besides, the percentages of $\mathrm{C} 2, \mathrm{C} 6$, and $\mathrm{C} 12$ monocytes were $9.7 \%$, $8.5 \%$, and $0.3 \%$, respectively in Gata $2^{\mathrm{WT} / \mathrm{WT}}-B C R / A B L$ vs $15.2 \%, 13.2 \%$, and $2.0 \%$, respectively in Gata2 $2^{\mathrm{WT} / \mathrm{L} 359 \mathrm{~V}}$ $B C R / A B L$ group (Fig. $7 d$ ). Our observation of the increase in the BM percentages of GMPs and monocytes suggested that the leukemic cell mass was, at least in part, directed to the monocytic precursors dysregulated by Gata2L359V mutation on the basis of $B C R / A B L$-induced CML $(30.3 \%$ vs $18.5 \%)$. When scRNA-seq data were subject to 
A

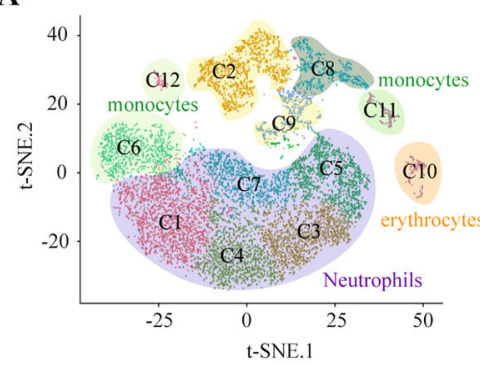

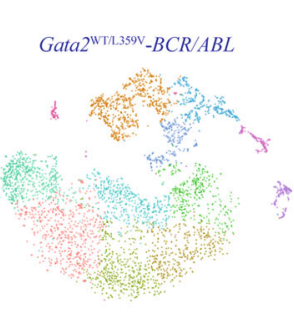

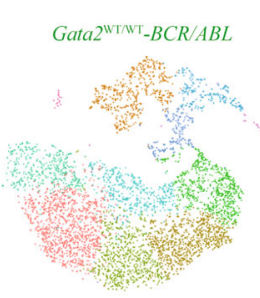

- C1 Neutrophil

- C2 Itgam ${ }^{\text {high }} L y 6 g^{\text {low }}$ Monocyte

- C3 Neutrophil

- C4 Neutrophil

- C5 Neutrophil

C6 CdI $4^{\text {high }} / \operatorname{tgam}^{\text {high }}$ Monocyte

- C7 Neutrophil

C8 $\mathrm{Mpo}^{+} \mathrm{Kit}^{+} \mathrm{GMP}$

C9 Ms4a3 ${ }^{+} \mathrm{Kit}^{+} \mathrm{GMP}$

C10 Erythroblast

- C11 Gata2 ${ }^{+}$it $^{+} \mathrm{GMP}$

- 12 Adgre1 $1^{+}$Csfl $r^{+}$Monocyte

B

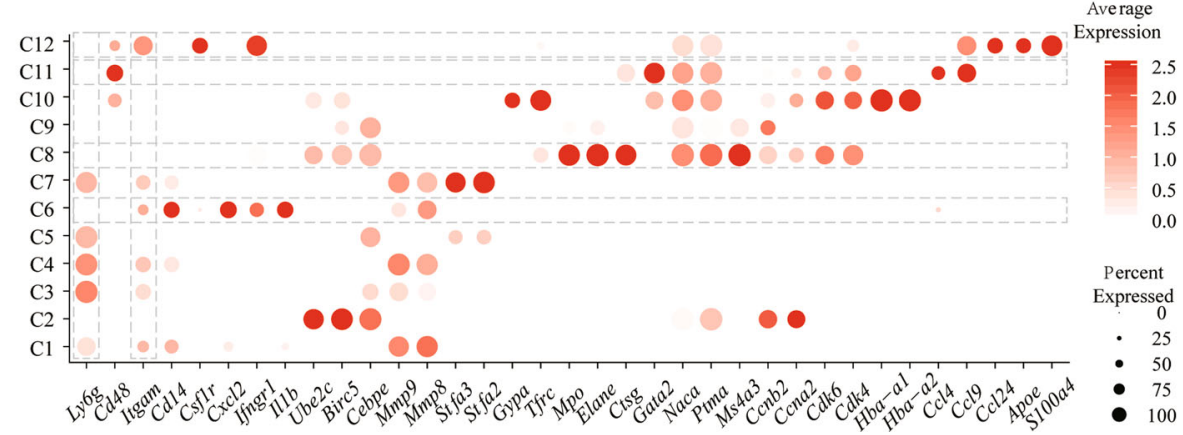

C
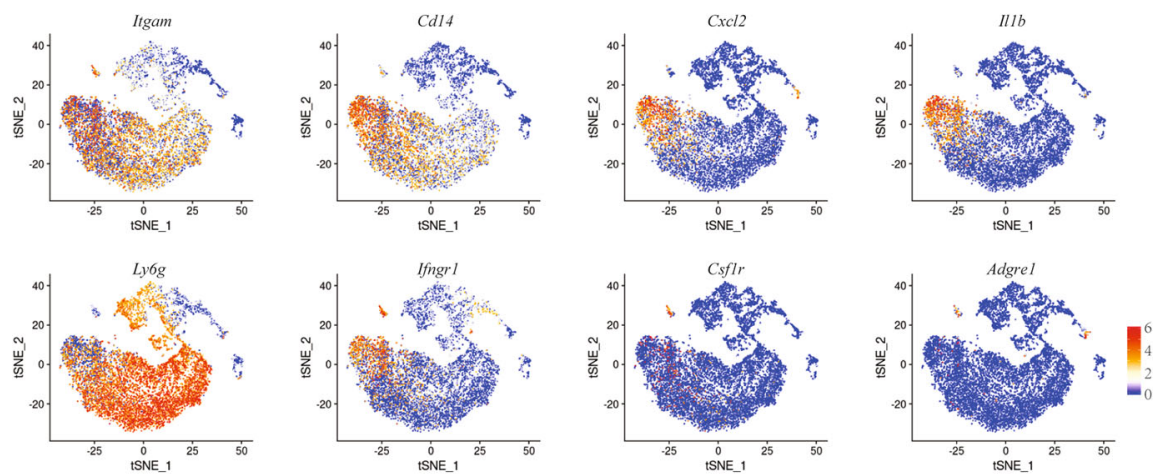

D

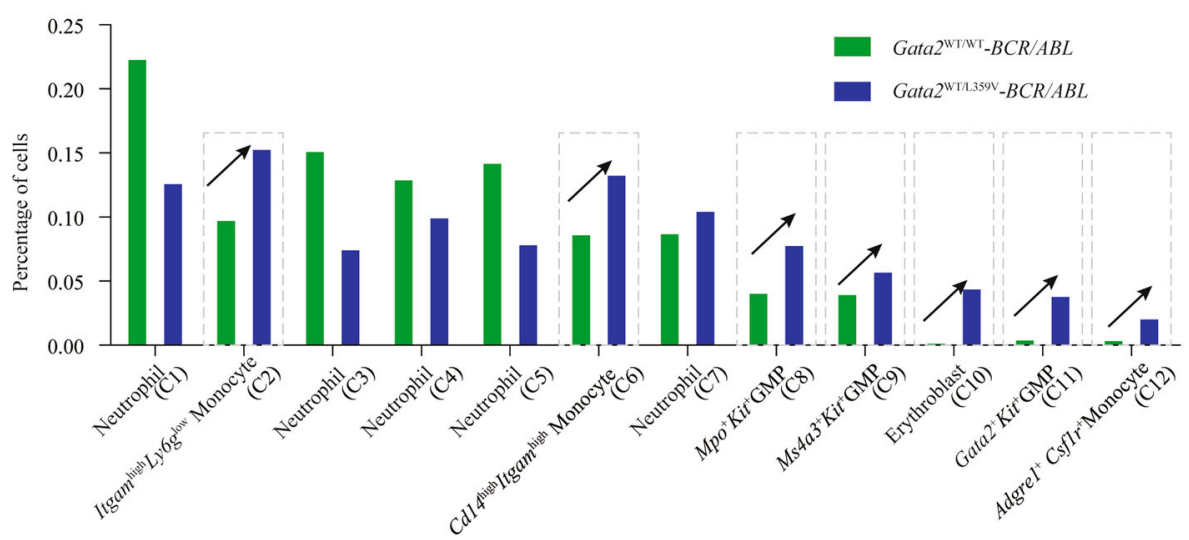

Fig. 7 Dynamic changes between leukemic cells of Gata $2^{\mathrm{WT} / L 359 \mathrm{v}}-B C R / A B L$ and $G a t a 2^{\mathrm{WT} / \mathrm{WT}}-B C R / A B L$ mice revealed by single-cell RNA-seq analysis. A t-distributed Stochastic Neighbor Embedding (t-SNE) showing 12 clusters of BM cells from Gata2 ${ }^{\text {WT/L359V }}-B C R / A B L$ and Gata2 ${ }^{\text {WT } / W T}-B C R /$ $A B L$ mice. The left panel showed the 12 clusters of the merged scRNA-seq datasets. The middle panels showed the separated illustration of the t-SNE

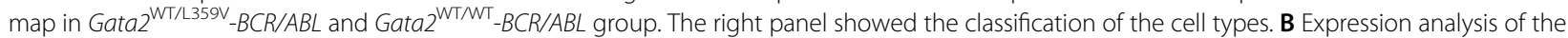
cluster-specific genes. Dot plot showing the expression of representative genes across the 12 classes of cells. $\mathbf{C}$ t-SNE showing the expression of representative genes in different classes of cells. D Comparison of the percentage of each class of cells between Gata2 ${ }^{\text {WT/L359V }}-B C R / A B L$ and Gata $2^{\text {WTMT }}-B C R$ $A B L B M$. 
further analysis, pathways related to myeloid/leukocyte differentiation and function were found dysregulated in GMPs of Gata $2^{\mathrm{WT} / \mathrm{L} 359 \mathrm{~V}}-B C R / A B L$ mouse (Fig. S8B). Moreover, Pu.1 targets were observed down-regulated in the neutrophils and $\mathrm{Csf1r^{+ }}$ monocytes of Gata $2^{\mathrm{WT} / \mathrm{L} 359 \mathrm{~V}}$. $B C R / A B L \mathrm{BM}$ (Fig. S9), supporting the interference of Pu.1 function upon the effect of Gata2-L359V as previously reported by biochemical approach ${ }^{25}$. These data suggested that Gata2-L359V partially impeded the cell differentiation at the early stage of myelomonocytic lineage, thus promoting the CML progression.

\section{Discussion}

GATA2 is a master zinc-finger transcription factor and plays essential roles in the regulation of HSC activity and stimulation of myeloid-erythroid progenitor differentiation under physiological conditions ${ }^{58,59}$. In the present work, we have provided further evidence for the pivotal function of Gata2 in the regulation of primitive and definitive hematopoiesis with a murine Gata2-L359V knockin model. RNA-seq data and integrative analysis of available gene expression datasets in relevant cell/tissue systems allowed us to address the molecular networks underlying the major phenotypic features at distinct development stages. Meanwhile, by using scRNA-seq, we showed that heterozygous Gata2-L359V mutation induced an increased number of GMPs associated with monocytic lineage expansion in $B C R / A B L$-transduced CML model.

It has been well established that primitive hematopoiesis is key to the murine embryonic development at E7.5 ${ }^{60,61}$. Embryos with defects of the primitive hematopoiesis cannot develop beyond E11.5. Then the definitive hematopoiesis, originated from aorta-gonad-mesonephros (AGM) region of the embryo, takes place in fetal liver and BM to ensure the functions of blood system through fetal life to after birth. The downregulation of Gata2 induced by gene $\mathrm{KO}$ or the deletion of a cis-regulatory element of Gata2 resulted in embryonic lethality with severe anemia at E10.5 ${ }^{10,58}$. Intriguingly, the Gata2 $2^{\mathrm{L} 359 \mathrm{~V} / \mathrm{L} 359 \mathrm{~V}}$ embryos showed a similar death outcome and deficient erythroid differentiation around E11.5. In concordance, genes involved in embryonic hematopoiesis and other development processes were dysregulated in Gata $2^{\mathrm{L} 359 \mathrm{~V} / \mathrm{L} 359 \mathrm{~V}}$ but not in Gata $2^{\mathrm{WT} / \mathrm{L} 359 \mathrm{~V}}$ yolk sac. Nevertheless, compared to Gata $2^{\mathrm{WT} / \mathrm{WT}}$, the Gata $^{\mathrm{WT} / \mathrm{L} 359 \mathrm{~V}}$ embryos still harbored distinct expression profiles of genes related to some important signal transduction, heralding a potential non-lethal defect in the heterozygous mutants.

Indeed, during adult life, although Gata2 ${ }^{\mathrm{WT} / \mathrm{L} 359 \mathrm{~V}}$ mice displayed no obvious abnormality under steady-state conditions, they showed dramatically reduced BM reconstitution capacity under stress, likely due to the exhaustion of the HSC quiescent pool. RNA-seq data showed that the expression of some transcription factors regulating hematopoietic differentiation was downregulated whereas the expression levels of genes promoting cell cycle and proliferation were increased in Gata2-L359V mutants, which could impair HSC and MPP functions. Application of serial BM transplantation experiment with improved technology in the future may further define the impact of Gata2-L359V on long-term HSCs. Growing evidence has demonstrated that mice lacking Gata2 exhibited a defect in definitive hematopoiesis $^{6,9}$, and the haploinsufficiency of GATA2 perturbs HSC homeostasis ${ }^{6,9,59}$. The overexpression of GATA2 also suppresses hematopoiesis ${ }^{11,62}$. Hence, the integrity of the Gata2 associated regulatory network is indispensable for a balanced self-renewal/differentiation potential of HSCs during adult hematopoiesis.

An interesting finding of the present work is the enhanced DNA-binding capacity of Gata2-L359V than Gata2-WT in ChIP-seq analysis. The core DNA binding preference of GATA2 is the 'GAT' motif, whereas the well-characterized DNA-binding site of GATA2 is the 'GATAA' motif identified in the regulation of erythropoietic differentiation ${ }^{63,64}$. In fact, the motifs of GATA2 may vary in distinct cell types. Different from the canonical GATAA motif, Gata2-WT and Gata2-L359V in $32 \mathrm{D}$ cells were found to preferentially bind to the 'GATG' repeat sequence, implying that the mechanism underlying the regulation of Gata2 on early hematopoietic differentiation in the context of 32D cell line might be slightly different from that on terminal erythropoietic differentiation. Taken together, our data suggest that the Gata2 level and appropriate target occupancy must be constrained within a physiological window, while its insufficient or excessive activity and/or scope could impair hematopoiesis ${ }^{65}$. On the other hand, Gata2-L359V should be considered as an aberrant transcription factor with complex functions instead of a simple "gain-of-function" mutation. A study focusing on the GATA switch is also warranted in the future for understanding the mechanism behind the dysregulation of Gata2-L359V on hematopoiesis at a distinct dimension.

The Gata2-L359V mutation has been initially identified by our group as an aberrant transcription factor in cooperation with $B C R / A B L$ fusion gene in CML patients with myelomonoblastic transformation ${ }^{25}$. By using murine BM transplantation model, Gata2-L359V, and BCR/ $A B L$ co-transduction led to an increase of $\mathrm{BM}$ monocytes $^{22}$. In contrast to the human course of CML and other murine AML model, CML-like disease induced by retroviral $B C R / A B L$ transfer in mice often deteriorates rapidly due to capillary embolism caused by excessive mature granulocytes in vital organs such as the lungs. Paradoxically, the burden of the peripheral embolism was relieved in CML blast-crisis mice by the blockage of cell 
differentiation, leading to a longer survival ${ }^{51-53}$. Consistent with this, Gata $2^{\mathrm{WT} / \mathrm{L} 359 \mathrm{~V}}-B C R / A B L$ mice also lived longer than the Gata2 $2^{\mathrm{WT} / \mathrm{WT}}-B C R / A B L$ controls. By performing scRNA-seq analysis, we identified 12 different t-distributed stochastic neighbor embedding (tSNE) clusters from leukemic cells based on their transcriptomic signature, which allowed the detection of subtle differences among distinct cell types at the molecular level. Flow cytometry or Cytometry by Time of Flight (CyTOF) analysis might be conducted to further validate the cell features in the future. Also, studies on the biological function of these subsets of cells might be conducted to explore the Gata2-L359V mediated abnormality in CML. Moreover, we found that Gata2L359V suppressed the expression of Pu.1 targets in neutrophils and $\mathrm{Csflr}^{+}$monocytes by using the scRNAseq data, supporting our previous in vitro study that Gata2 L359 could interfere with Pu.1 function. These results have further enriched our understanding of the activity of Gata2-L359V in blocking the differentiation of $B C R / A B L$-expressing CML stem/progenitor cells, thus helping us comprehend the stepwise pathogenesis in this unique disease model.

\section{Materials and methods Mice experiments}

All animal experiments were conducted following the institutional ethical guidelines on animal care and approved by the Department of Animal Experimentation of the Shanghai Jiao Tong University School of Medicine. The Gata2-L359V knockin murine model was constructed by the Model Animal Research Center of Nanjing University, China. The L359V mutation was introduced into the ZF2 domain located in the exon 5 of the murine Gata2 gene with a PGK-neo cassette in the nearby intron (Figs. 1a and S1A). The Gata2 target fragment was cloned into the pMD-18T vector, followed by point mutagenesis of L359V. Meanwhile, the Gata2 target fragment on the BAC plasmid was replaced by the rpsL-neo cassette using gene recombination, and then the rpsL-neo cassette was replaced by the nonselectable fragment containing L359V mutation. The fragment containing the $5 \mathrm{~kb} 5^{\prime}$ arm, mutation point, and $5 \mathrm{~kb} 3^{\prime}$ arm was retrieved to the retrieving vector PL253 by gene recombination, and the PGK-neo cassette was subsequently inserted into the intron near the mutation point as a selective marker. The PL253 vector was then linearized at the $5^{\prime}$ end and electroporated into ES cells. G418-resistant ES cell clones were identified using Southern blot and injected into blastocysts to generate chimeric mice. Allele-specific primer sets were designed to distinguish between wild-type and mutated genotypes and were listed in Table S5. All experiments were performed in C57BL/6 mice except the CML model, which was built in mice on BALB/c background through backcross breeding.

\section{Embryo morphology}

Yolk sacs were fixed in methylcellulose and observed under a stereomicroscope (Nikko ECLIPSE TS100). The survival of embryos was determined by heartbeat or embryo dissolution. The PECAM-1 staining was performed using monoclonal antibody MEC13.3 and detected using an HRP Detection Kit (BD Bioscience). Hemoglobin was stained with $o$-dianisidine (SigmaAldrich). The erythrocytes from the yolk sacs were stained using Wright's stain (Sigma-Aldrich) and photographed under a microscope (Olympus BX61TRF).

\section{5-FU treatment}

Gata $2^{\mathrm{WT} / \mathrm{WT}}$ and Gata2 ${ }^{\mathrm{WT} / \mathrm{L} 359 \mathrm{~V}}$ mice aged 6-8 weeks received a single intraperitoneal injection of $150 \mathrm{mg} / \mathrm{kg} \mathrm{5-}$ FU (Sigma-Aldrich). The PB mononuclear cells were counted every three days. Additionally, Gata2 $2^{\mathrm{WT} / \mathrm{WT}}$ and Gata $^{\mathrm{WT} / \mathrm{L} 359 \mathrm{~V}}$ mice were injected with $150 \mathrm{mg} / \mathrm{kg} 5-\mathrm{FU}$ every 10 days for survival curve analysis.

\section{Competitive BM transplantation}

A total of 40 male C57BL/6 mice aged 6-8 weeks were used in competitive transplantation. The BM cells isolated from CD45.2 mice $\left(5 \times 10^{5}\right.$ cells $)$ were mixed with an equivalent number of cells from CD45.1 mice and transplanted into lethally irradiated CD45.1 WT recipients. The percentage of CD45.2 $2^{+}$cells in $\mathrm{PB}$ of the engrafted recipients were tested every 4 weeks, and BM cells were harvested at 16 weeks after transplantation. The immunophenotype of BM cells was analyzed via flow cytometry by using the mouse lineage antibody cocktail and antibodies against c-Kit, Sca-1, CD150, CD48, CD16, CD32, CD34, and CD45.2. Cells were stained, fixed, and permeated following the manufacturer's instruction of the Transcription Factor Buffer Set (BD Pharmingen ${ }^{\mathrm{TM}}$, 562574).

\section{Flow cytometry, western blot, and real-time qPCR experiments}

Flow cytometry was performed on the FACSLSRII flow cytometers (BD Biosciences) and analyzed using the FlowJo Software (version 9.3.2). All antibodies were purchased from BD Biosciences. The western blot experiments were described as previously described ${ }^{66,67}$. Antibodies against the $\mathrm{N}$-terminals of Gata2 were used for the detection of the embryonic Gata2 expression. Antibodies against Flag were used for the detection of Flagtagged Gata2 proteins in 32D cells. Antibodies against Gapdh were used as the internal control. RT-qPCR was performed to validate the RNA-seq results. Total RNA was extracted as described above, and cDNA was synthesized using the PrimeScript ${ }^{\circledR}$ RT reagent Kit (TaKaRa Biotechnology Co. Ltd.). Real-time qPCR was performed using the primers listed in Table S5. 


\section{Retroviral transduction and BM transplantation}

Retrovirus generation and BM transplantation were performed as previously described ${ }^{51,52}$. Briefly, a total of 50 male $\mathrm{BALB} / \mathrm{c}$ mice aged 6-8 weeks were used in retroviral transduction and $\mathrm{BM}$ transplantation. $\mathrm{BM}$ cells were isolated from the donor mice pretreated with 5 -FU $(250 \mathrm{mg} / \mathrm{kg})$ and infected with retroviruses containing MigR1-BCR/ABL once daily for 2 days in transplant medium. In all, $5 \times 10^{5}$ cells per mice were transplanted into the irradiated (3.4 Gy twice at a 3-h interval) recipient mice through tail vein injection randomly. After 3 weeks of transplantation, BM cells were subjected to morphological examination, flow cytometry analysis, and scRNA-seq analysis. For morphological examination, cells were centrifuged onto a glass slide and subjected to Wright's staining (Sigma-Aldrich). Light microscopy images were obtained using the Nikko ECLIPSE TS100.

\section{RNA-seq and data analysis}

The RNA-seq experiments were conducted as previously reported $^{67}$. E9.5 yolk sacs dissected under a microscope were used for RNA-seq analysis. For each genotype (Gata $2^{\text {WT/WT }}$, Gata $2^{\mathrm{WT} / \mathrm{L} 359 \mathrm{~V}}$, and Gata $\left.^{\mathrm{L} 359 \mathrm{~V} / \mathrm{L} 359 \mathrm{~V}}\right)$, three yolk sacs were used as biological replicates. The DNA and RNA were simultaneously isolated with the All Prep DNA/RNA Mini Kit (Qiagen) according to the manufacture's instruction. The DNA sample was sent for genotyping and RNA samples were used for RNA-seq analysis. LSK cells isolated from the BM of 6- to 8-week-old Gata $2^{\text {WT/WT }}$ and Gata $2^{\mathrm{WT} / \mathrm{L} 359 \mathrm{~V}}$ male mice were used for RNA isolation. Three biological replicates were carried out for each genotype $\left(\right.$ Gata $2^{\mathrm{WT} / \mathrm{WT}}$ and $\left.G a t a 2^{\mathrm{WT} / \mathrm{L} 359 \mathrm{~V}}\right)$, and three individual $\mathrm{BM}$ of the same genotype were mixed as one biological sample. RNA-sequencing libraries were constructed with the SMARTer ${ }^{\circledR}$ Universal Low Input RNA Kit for Sequencing according to the manufacture's instruction. The libraries were sequenced with the Illumina MiSeq.

The RNA-seq data in FASTQ format was mapped against the mouse genome $(\mathrm{mm} 10)$ using the STAR $(\mathrm{v} 2.7 .0)^{68}$, and the counts of each gene were calculated using HTseq (v0.6.1) ${ }^{69}$. The gene annotation files were downloaded from the UCSC Genome Browser (http:// hgdownload.soe.ucsc.edu/). The significantly differentially expressed genes between different conditions were obtained using the DEseq $2^{70}$ with a cutoff of the adjusted $p$-value (FDR) $<0.05$ and $\mid \log _{2}$ (Fold Change) $\mid>0.58$. The fragments per kilobase million (FPKM) were used to evaluate gene expression levels by normalizing the length of genes using the count matrix. The $\mathrm{R}$ package limma ${ }^{71}$ was used to identify differentially expressed genes when the input was an FPKM matrix (Expression profiles retrieved from the GEO database).

\section{ChIP-Seq and data analysis}

The ChIP-seq experiments were conducted as previously described ${ }^{67}$. The full length of Gata2-WT and Gata2-L359V were cloned into the MigR1-Flag plasmid vector and transfected into $32 \mathrm{D}$ cells. The anti-Flag antibody was used in the ChIP experiments. The ChIPseq DNA libraries were constructed using the VAHTS Universal Pro DNA Library Prep Kit (Vazyme, Nanjing, China) according to the manufacturer's instructions. The libraries were sequenced on the NovaSeq 6000. For ChIPseq data analysis, all sequencing reads were mapped against the mouse genome $(\mathrm{mm} 10)$ by using the bowtie 2 (version 2.3.0) ${ }^{72}$ and uniquely mapped reads were kept for downstream analysis. The high confident binding peaks were called by overlapping peaks identified by the MACS suite (version 1.4.3) ${ }^{73}$ and the Homer Suite ${ }^{74}$. Visualization of the peaks was performed in the UCSC genome browser. The motif enrichment analysis was performed using the MEME suite (version 4.11.1) ${ }^{75}$. For GATAswitch analysis, the target genes of GATA1 and GATA2 were obtained from Fujiwara et al. ${ }^{64}$ (Table S6).

\section{Functional and pathway enrichment analysis of expression profiling data}

GO enrichment analysis of differentially expressed genes between different groups was performed using both DAVID (https://david.ncifcrf.gov/) and STRING (https:// string-db.org/) with the default parameters. Normalized RNA-seq data were rank-ordered by the fold change of gene expression between different groups. To identify the enriched pathways, GSEA ${ }^{76}$ was performed using $R$ package clusterProfiler ${ }^{77}$. Gene sets enrolled in the study were downloaded from two databases, the Molecular Signatures Database (MSigDB) of the Broad Institute, and the Mammalian Phenotype (MP) Ontology ${ }^{26}$. HALLMARK gene sets $(\mathrm{H})$ and MSigDB curated gene sets $(\mathrm{C} 2$, C5) were used to perform GSEA analysis ${ }^{76}$. Gene IDs transformation between human and mouse was performed using R package biomaRt (https://bioconductor. org/packages/biomaRt/). R package enrichplot (https:// github.com/GuangchuangYu/enrichplot) was used to interpret enrichment results of GSEA. For integration analysis with other Gata2 hematopoietic mouse models, the gene expression profiling of Gata2 KO and Gata2 WT was collected from Gene Expression Omnibus (GEO) with accession id GSE133248 ${ }^{40}$. Gene markers of murine hematopoietic cells (e.g., LT-HSC, ST-HSC, and MPP) were obtained from Haemopedia database (https:// haemosphere.org/), which was published by Graaf et al. ${ }^{78}$.

\section{scRNA-seq and data analysis}

$\mathrm{GFP}^{+} \mathrm{BM}$ cells were harvested from leukemic Gata $2^{\mathrm{WT} / \mathrm{L} 359 \mathrm{~V}}-B C R / A B L$ and Gata2 $2^{\mathrm{WT} / \mathrm{WT}}-B C R / A B L$ mice and loaded onto a GemCode Single-Cell Instrument 
(10X Genomics, Pleasanton, CA, USA). Single-cell RNAseq libraries were constructed using the Chromium Single-cell 3' Library Kit (10X genomics) and analyzed on the Illumina NovaSeq 6000. The 10X Genomics cell ranger v2.1.1 was used for raw sequence alignment, filtering, barcode counting, and unique molecular identifier (UMI) counting. The gene-cell-barcode count matrix was analyzed using the R Seurat (v3.1.2) package ${ }^{79}$. Cells that expressed less than 500 genes or over $10 \%$ mitochondrial RNA were filtered out. Genes that expressed in less than $0.1 \%$ of total cells were removed. The normalization method was used to normalize the filtered gene expression count matrix using the default parameters and workflows provided by Seurat ${ }^{79}$. The expression data were merged and integrated using Seurat, and 2000 variable genes were identified for batch effect correction. A total of 4844 cells and 2943 cells were obtained from the Gata2 $2^{\mathrm{WT} / \mathrm{WT}}-B C R / A B L$ and Gata2 $2^{\mathrm{WT} / \mathrm{L} 359 \mathrm{~V}}-B C R / A B L$ mice, respectively. For visualization, tSNE was used ${ }^{80}$. Cell types in each cluster was identified referred to the top markers with adjusted $p$-value (p_val_adj) $\leq 0.05$ and average log fold change (avg_logFC) $\geq 0.5$. The lineages were identified by the top-expressed cluster-specific genes. The lineage-specific genes were selected according to the previous reports ${ }^{54,55}$.

\section{Statistical analysis}

The Student unpaired two-tailed $t$-test was used for group comparisons. Differences were considered significant at $p<0.05$. To decrease false-positive rates, we used FDR correction in multiple test analysis. $\mathrm{R}$ package ggplot2 and pheatmap were used for visualization. All statistical analyses were performed using the GraphPad Prism software (GraphPad Software, San Diego, CA) and R software (version 4.0.2, http://www.R-project.org).

\section{Data sharing statement}

All sequencing data included in this study are available at Sequence Read Archive (SRA) database (PRJNA659109).

\section{Acknowledgements}

We would like to thank our colleagues in Shanghai Institute of Hematology for their support and discussion. We thank Dr. Hai Fang in National Research Center for Translational Medicine (Shanghai) for his constructive advice.

\footnotetext{
Author details

${ }^{1}$ Shanghai Institute of Hematology, State Key Laboratory of Medical Genomics, National Research Center for Translational Medicine, Ruijin Hospital Affiliated to Shanghai Jiao Tong University (SJTU) School of Medicine, Shanghai, China. ${ }^{2}$ Institute of Health Sciences, Shanghai Institutes for Biological Sciences and Graduate School, Chinese Academy of Sciences and SJTU School of Medicine, Shanghai, China. ${ }^{3}$ Cancer Research Center, Sheba Medical Center, Sackler Faculty of Medicine, Tel Aviv University, Tel Aviv, Israel. ${ }^{4}$ Division of Pediatric Hemato-Oncology, Schneider Children's Medical Center of Israel, Sackler Faculty of Medicine, Tel Aviv University, Tel Aviv, Israel. ${ }^{5}$ Present address: Department of Rheumatology, Renji Hospital Affiliated to Shanghai Jiao Tong University School of Medicine, Shanghai, China
}

\section{Author contributions}

Q.H.H., Z.C., and S.J.C. designed research; Y.K.F., Y.T., X.G.X., Z.W.C., B.W., M.M.P. J.W., L.J., Y.Y.X., J.L., P.L., X.P.L., M.L.Z., Q.W., and Q.H.H. performed experiments Y.T., Y.T.D., N.Q., and J.Y.H. performed bioinformatics analysis. Y.T., Y.K.F., Z.W.C. B.W., M.M.P., Y.L.Z., B.C., A.R., S.I., X.J.S., J.Y.H., Q.H.H., Z.C., and S.J.C. analyzed data. Y.K.F., Y.T., B.W., M.M.P., Z.W.C., Q.H.H., Z.C. and S.J.C. wrote the manuscript.

\section{Funding}

This work was supported in part by the Chinese National Key Basic Research Project (973, 2013CB966801); the Chinese Ministry of Health (201202003); the Overseas Expertise Introduction Project for Discipline Innovation (111 Project, B17029); Shanghai Collaborative Innovation Program on Regenerative Medicine and Stem Cell Research (2019CXJQ01); Double First-Class Project (WF510162602) from Shanghai Jiaotong University; National Natural Science Foundation of China (81170464); Joint NSFC (National Natural Science Foundation of China)-ISF (Israel Science Foundation) Grant (81461148023); and the Samuel Waxman Cancer Research Foundation.

\section{Conflict of interest}

The authors declare no competing interests.

\section{Ethical approval}

All animal experiments were conducted following the institutional ethical guidelines on animal care and approved by the Department of Animal Experimentation of the Shanghai Jiao Tong University School of Medicine.

\section{Publisher's note}

Springer Nature remains neutral with regard to jurisdictional claims in published maps and institutional affiliations.

Supplementary information The online version contains supplementary material available at https://doi.org/10.1038/s41419-021-03826-1.

Received: 30 October 2020 Revised: 1 May 2021 Accepted: 11 May 2021 Published online: 02 June 2021

\section{References}

1. Orkin, S. H. Diversification of haematopoietic stem cells to specific lineages. Nat. Rev. Genet. 1, 57-64 (2000).

2. Zhang, $X$. et al. DNMT3A and TET2 compete and cooperate to repress lineagespecific transcription factors in hematopoietic stem cells. Nat. Genet. 48, 1014-1023 (2016).

3. Cancer Genome Atlas Research Network et al. Genomic and epigenomic landscapes of adult de novo acute myeloid leukemia. N. Engl. J. Med. 368, 2059-2074 (2013)

4. Vicente, C., Conchillo, A., Garcia-Sanchez, M. A. \& Odero, M. D. The role of the GATA2 transcription factor in normal and malignant hematopoiesis. Crit. Rev. Oncol. Hematol. 82, 1-17 (2012).

5. Ling, T. et al. Chromatin occupancy and epigenetic analysis reveal new insights into the function of the GATA1 $\mathrm{N}$ terminus in erythropoiesis. Blood 134, 1619-1631 (2019).

6. Tsai, F. Y. et al. An early haematopoietic defect in mice lacking the transcription factor GATA-2. Nature 371, 221-226 (1994).

7. Horwitz, M. S. GATA2 deficiency: flesh and blood. Blood 123, 799-800 (2014).

8. Gao, X. et al. Gata2 cis-element is required for hematopoietic stem cell generation in the mammalian embryo. J. Exp. Med. 210, 2833-2842 (2013).

9. de Pater, E. et al. Gata2 is required for HSC generation and survival. J. Exp. Med. 210, 2843-2850 (2013).

10. Johnson, K. D. et al. Cis-element mutated in GATA2-dependent immunodeficiency governs hematopoiesis and vascular integrity. J. Clin. Investig. 122, 3692-3704 (2012).

11. Tipping, A. J. et al. High GATA-2 expression inhibits human hematopoietic stem and progenitor cell function by effects on cell cycle. Blood $\mathbf{1 1 3}$, 2661-2672 (2009)

12. Rodrigues, N. P. et al. Haploinsufficiency of GATA-2 perturbs adult hematopoietic stem-cell homeostasis. Blood 106, 477-484 (2005). 
13. Dickinson, R. E. et al. Exome sequencing identifies GATA-2 mutation as the cause of dendritic cell, monocyte, B and NK lymphoid deficiency. Blood $\mathbf{1 1 8}$ 2656-2658 (2011).

14. Hsu, A. P. et al. Mutations in GATA2 are associated with the autosomal dominant and sporadic monocytopenia and mycobacterial infection (MonoMAC) syndrome. Blood 118, 2653-2655 (2011).

15. Greif, P. A. et al. GATA2 zinc finger 1 mutations associated with biallelic CEBPA mutations define a unique genetic entity of acute myeloid leukemia. Blood 120, 395-403 (2012).

16. Hahn, C. N. et al. Heritable GATA2 mutations associated with familial myelodysplastic syndrome and acute myeloid leukemia. Nat. Genet. 43, 1012-1017 (2011).

17. Fadilah, S. A., Cheong, S. K., Roslan, H., Rozie-Hanisa, M. \& Yen, G. K. GATA-1 and GATA-2 gene expression is related to the severity of dysplasia in myelodysplastic syndrome. Leukemia 16, 1563-1565 (2002).

18. Luesink, M. et al. High GATA2 expression is a poor prognostic marker in pediatric acute myeloid leukemia. Blood 120, 2064-2075 (2012).

19. Collin, M., Dickinson, R. \& Bigley, V. Haematopoietic and immune defects associated with GATA2 mutation. Br. J. Haematol. 169, 173-187 (2015).

20. Kazenwadel, J. et al. Loss-of-function germline GATA2 mutations in patients with MDS/AML or MonoMAC syndrome and primary lymphedema reveal a key role for GATA2 in the lymphatic vasculature. Blood 119 1283-1291 (2012).

21. Chong, C. E. et al. Differential effects on gene transcription and hematopoietic differentiation correlate with GATA2 mutant disease phenotypes. Leukemia $\mathbf{3 2}$ 194-202 (2018).

22. Zhang, S. J. et al. Gain-of-function mutation of GATA-2 in acute myeloid transformation of chronic myeloid leukemia. Proc. Natl Acad. Sci. USA 105, 2076-2081 (2008).

23. Tien, F. M. et al. GATA2 zinc finger 1 mutations are associated with distinct clinico-biological features and outcomes different from GATA2 zinc finger 2 mutations in adult acute myeloid leukemia. Blood Cancer J. 8, 87 (2018).

24. Basu, P. et al. EKLF and KLF2 have compensatory roles in embryonic betaglobin gene expression and primitive erythropoiesis. Blood 110, 3417-3425 (2007).

25. Carbon, S. et al. AmiGO: online access to ontology and annotation data. Bioinformatics 25, 288-289 (2009).

26. Smith, C. L. \& Eppig, J. T. The mammalian phenotype ontology: enabling robust annotation and comparative analysis. Wiley Interdiscip. Rev. Syst. Biol. Med. 1, 390-399 (2009).

27. lacobucci, l., et al. Modeling and targeting of erythroleukemia by hematopoietic genome editing. Blood 137, 1628-1640 (2021).

28. Lulli, V. et al. Overexpression of Ets-1 in human hematopoietic progenitor cells blocks erythroid and promotes megakaryocytic differentiation. Cell Death Differ. 13, 1064-1074 (2006).

29. Redondo Monte, E. et al. ZBTB7A prevents RUNX1-RUNX1T1-dependent clonal expansion of human hematopoietic stem and progenitor cells. Oncogene 39, 3195-3205 (2020).

30. Gekas, C. \& Graf, T. CD41 expression marks myeloid-biased adult hematopoietic stem cells and increases with age. Blood 121, 4463-4472 (2013).

31. Mitroulis, I. et al. Modulation of myelopoiesis progenitors is an integral component of trained immunity. Cell 172, 147-161.e112 (2018).

32. Taylor, S. J., et al. Preventing chemotherapy-induced myelosuppression by repurposing the FLT3 inhibitor quizartinib. Sci. Transl. Med. 9 eaam8060 (2017).

33. Oguro, H., Ding, L. \& Morrison, S. J. SLAM family markers resolve functionally distinct subpopulations of hematopoietic stem cells and multipotent progenitors. Cell Stem Cell 13, 102-116 (2013).

34. Li, Q. et al. Oncogenic Nras has bimodal effects on stem cells that sustainably increase competitiveness. Nature 504, 143-147 (2013).

35. Li, Y. et al. Single-cell analysis of neonatal HSC ontogeny reveals gradual and uncoordinated transcriptional reprogramming that begins before birth. Cell Stem Cell 27, 732-747.e737 (2020).

36. Staber, P. B. et al. Sustained PU.1 levels balance cell-cycle regulators to prevent exhaustion of adult hematopoietic stem cells. Mol. Cell 49 934-946 (2013).

37. Moran-Crusio, $\mathrm{K}$. et al. Tet2 loss leads to increased hematopoietic stem cell self-renewal and myeloid transformation. Cancer Cell 20, 11-24 (2011).

38. Jeong, M. et al. Loss of Dnmt3a immortalizes hematopoietic stem cells in vivo. Cell Rep. 23, 1-10 (2018)

39. Calvanese, V. et al. MLLT3 governs human haematopoietic stem-cell selfrenewal and engraftment. Nature 576, 281-286 (2019).
40. Menendez-Gonzalez, J. B. et al. Gata2 as a crucial regulator of stem cells in adult hematopoiesis and acute myeloid leukemia. Stem Cell Rep. 13, 291-306 (2019).

41. Olweus, J., Terstappen, L. W., Thompson, P. A. \& Lund-Johansen, F. Expression and function of receptors for stem cell factor and erythropoietin during lineage commitment of human hematopoietic progenitor cells. Blood $\mathbf{8 8}$, 1594-1607 (1996).

42. Bamford, R. N. et al. The interleukin (IL) 2 receptor beta chain is shared by IL-2 and a cytokine, provisionally designated IL-T, that stimulates T-cell proliferation and the induction of lymphokine-activated killer cells. Proc. Natl Acad. Sci. USA 91, 4940-4944 (1994).

43. Kanki, Y. et al. Epigenetically coordinated GATA2 binding is necessary for endothelium-specific endomucin expression. EMBO J. 30, 2582-2595 (2011).

44. Kobayashi-Osaki, M. et al. GATA motifs regulate early hematopoietic lineagespecific expression of the Gata2 gene. Mol. Cell Biol. 25, 7005-7020 (2005).

45. Lee, R., Kertesz, N., Joseph, S. B., Jegalian, A. \& Wu, H. Erythropoietin (Epo) and EpoR expression and 2 waves of erythropoiesis. Blood 98, 1408-1415 (2001).

46. Ghaleb, A. M. \& Yang, V. W. Kruppel-like factor 4 (KLF4): What we currently know. Gene 611, 27-37 (2017).

47. Kioussis, D. Aiolos: an ungrateful member of the Ikaros family. Immunity $\mathbf{2 6}$ 275-277 (2007).

48. Welinder, E., Ahsberg, J. \& Sigvardsson, M. B-lymphocyte commitment: identifying the point of no return. Semin. Immunol. 23, 335-340 (2011).

49. Fujiwara, $\mathrm{T}$. et al. Discovering hematopoietic mechanisms through genomewide analysis of GATA factor chromatin occupancy. Mol. Cell 36, 667-681 (2009).

50. Zhang, S. J., Shi, J. Y. \& Li, J. Y. GATA-2 L359 V mutation is exclusively associated with CML progression but not other hematological malignancies and GATA-2 P250A is a novel single nucleotide polymorphism. Leuk. Res. 33, 1141-1143 (2009).

51. Cuenco, G. M. \& Ren, R. Cooperation of BCR-ABL and AML1/MDS1/EVI1 in blocking myeloid differentiation and rapid induction of an acute myelogenous leukemia. Oncogene 20, 8236-8248 (2001).

52. Zhao, L. J. et al. Functional features of RUNX1 mutants in acute transformation of chronic myeloid leukemia and their contribution to inducing murine fullblown leukemia. Blood 119, 2873-2882 (2012).

53. Ren, R. Mechanisms of $B C R-A B L$ in the pathogenesis of chronic myelogenous leukaemia. Nat. Rev. Cancer 5, 172-183 (2005).

54. Han, X. et al. Mapping the mouse cell atlas by microwell-seq. Cell 173, 1307 (2018).

55. Nestorowa, S. et al. A single-cell resolution map of mouse hematopoietic stem and progenitor cell differentiation. Blood 128, e20-e31 (2016).

56. Paul, F. et al. Transcriptional heterogeneity and lineage commitment in myeloid progenitors. Cell 163, 1663-1677 (2015)

57. Han, X. et al. Mapping the mouse cell atlas by microwell-seq. Cell 172, 1091-1107.e1017 (2018).

58. Ling, K. W. et al. GATA-2 plays two functionally distinct roles during the ontogeny of hematopoietic stem cells. J. Exp. Med. 200, 871-882 (2004).

59. Rodrigues, N. P. et al. Haploinsufficiency of GATA-2 perturbs adult hematopoietic stem-cell homeostasis. Blood 106, 477-484 (2005).

60. Orkin, S. H. GATA-binding transcription factors in hematopoietic cells. Blood 80, 575-581 (1992).

61. Ferkowicz, M. J. \& Yoder, M. C. Blood island formation: longstanding observations and modern interpretations. Exp. Hematol. 33, 1041-1047 (2005)

62. Persons, D. A. et al. Enforced expression of the GATA-2 transcription factor blocks normal hematopoiesis. Blood 93, 488-499 (1999).

63. Dore, L. C., Chlon, T. M., Brown, C. D., White, K. P. \& Crispino, J. D. Chromatin occupancy analysis reveals genome-wide GATA factor switching during hematopoiesis. Blood 119, 3724-3733 (2012).

64. Fujiwara, T. et al. Discovering hematopoietic mechanisms through genomewide analysis of GATA factor chromatin occupancy. Mol. Cell 36, 667-681 (2009).

65. Katsumura, K. R. et al. Human leukemia mutations corrupt but do not abrogate GATA-2 function. Proc. Natl Acad. Sci. USA 115, E10109-E10118 (2018).

66. Chen, B. Y. et al. SETD2 deficiency accelerates MDS-associated leukemogenesis via S100a9 in NHD13 mice and predicts poor prognosis in MDS. Blood 135 , 2271-2285 (2020)

67. Tan, Y. et al. A PML/RARalpha direct target atlas redefines transcriptional deregulation in acute promyelocytic leukemia. Blood 137, 1503-1516 (2020).

68. Dobin, A. et al. STAR: ultrafast universal RNA-seq aligner. Bioinformatics 29 15-21 (2013). 
69. Anders, S., Pyl, P. T. \& Huber, W. HTSeq-a Python framework to work with high-throughput sequencing data. Bioinformatics 31, 166-169 (2015).

70. Love, M. I., Huber, W. \& Anders, S. Moderated estimation of fold change and dispersion for RNA-seq data with DESeq2. Genome Biol. 15, 550 (2014).

71. Ritchie, M. E. et al. limma powers differential expression analyses for RNA-sequencing and microarray studies. Nucleic Acids Res. 43, e47 (2015).

72. Langmead, B. \& Salzberg, S. L. Fast gapped-read alignment with Bowtie 2. Nat. Methods 9, 357-359 (2012)

73. Zhang, Y. et al. Model-based analysis of ChIP-Seq (MACS). Genome Biol. 9 R137 (2008).

74. Heinz, S. et al. Simple combinations of lineage-determining transcription factors prime cis-regulatory elements required for macrophage and B cel identities. Mol. Cell 38, 576-589 (2010).
75. Bailey, T. L. et al. MEME SUITE: tools for motif discovery and searching. Nucleic Acids Res. 37, W202-W208 (2009).

76. Subramanian, A. et al. Gene set enrichment analysis: a knowledge-based approach for interpreting genome-wide expression profiles. Proc. Natl Acad. Sci. USA 102, 15545-15550 (2005).

77. Yu, G., Wang, L. G., Han, Y. \& He, Q. Y. clusterProfiler: an R package for comparing biological themes among gene clusters. OMICS 16, 284-287 (2012).

78. de Graaf, C. A. et al. Haemopedia: an expression atlas of murine hematopoietic cells. Stem Cell Rep. 7, 571-582 (2016).

79. Butler, A., Hoffman, P., Smibert, P., Papalexi, E. \& Satija, R. Integrating single-cell transcriptomic data across different conditions, technologies, and species. Nat. Biotechnol. 36, 411-420 (2018)

80. Amir el, A. D. et al. viSNE enables visualization of high dimensional single-cel data and reveals phenotypic heterogeneity of leukemia. Nat. Biotechnol. $\mathbf{3 1}$ 545-552 (2013). 